\title{
Single point gradient blow-up on the boundary for a Hamilton-Jacobi equation with $p$-Laplacian diffusion
}

\author{
Amal Attouchi and Philippe Souplet
}

\begin{abstract}
We study the initial-boundary value problem for the Hamilton-Jacobi equation with nonlinear diffusion $u_{t}=\Delta_{p} u+|\nabla u|^{q}$ in a two-dimensional domain for $q>$ $p>2$. It is known that the spatial derivative of solutions may become unbounded in finite time while the solutions themselves remain bounded. We show that, for suitably localized and monotone initial data, the gradient blow-up occurs at a single point of the boundary. Such a result was known up to now only in the case of linear diffusion $(p=2)$. The analysis in the case $p>2$ is considerably more delicate.
\end{abstract}




\section{Contents}

1 Introduction

1.1 Problem and main result . . . . . . . . . . . . . . . . . 3

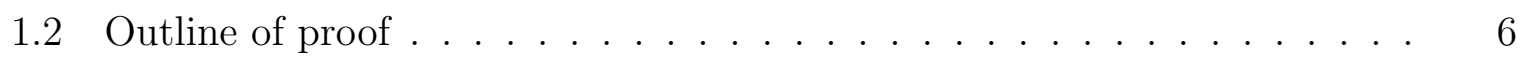

2 Local well-posedness and regularity

3 Preliminary estimates: $x$-symmetry, lower bound on $u_{y}$ and bound on $u_{t}$

4 Finite-time gradient blow-up for concentrated initial data 12

5 Local boundary control for the gradient and localization of the gradient blow-up set

6 Existence of well-prepared initial data: proof of Theorem 1.1(i) 16

7 Nondegeneracy of gradient blow-up points 17

8 The auxiliary function $J$ and the proof of single-point gradient blow-up $\mathbf{1 9}$

8.1 Parabolic inequality for the auxiliary function $J \ldots \ldots \ldots$

8.2 Boundary conditions for the auxiliary function $J \ldots \ldots \ldots$. . . . . 21

8.3 Initial conditions for $J \ldots \ldots \ldots \ldots \ldots \ldots$

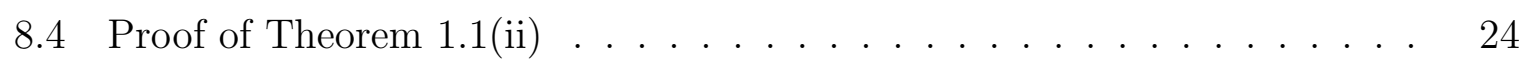

9 Proof of the main parabolic inequality (Proposition 8.1) 24

10 APPENDIX 1. Proof of regularity results (Theorem 2.2) 37

11 APPENDIX 2. A parabolic version of Serrin's corner lemma 39 


\section{Introduction}

\subsection{Problem and main result}

This article is a contribution to the study of the influence of nonlinear diffusion on the qualitative properties of equations of Hamilton-Jacobi type and, in particular, on the formation of finite-time singularity. More specifically, we consider the following problem

$$
\begin{cases}u_{t}=\Delta_{p} u+|\nabla u|^{q}, & (x, y) \in \Omega, \quad t>0, \\ u(x, y, t)=\mu y, & (x, y) \in \partial \Omega, \quad t>0, \\ u(x, y, 0)=u_{0}(x, y), & (x, y) \in \Omega,\end{cases}
$$

where $\Delta_{p}$ denotes the $p$-Laplace operator, $\Delta_{p}=\nabla \cdot\left(|\nabla u|^{p-2} \nabla u\right)$. Throughout this paper, we assume that $\mu \geq 0$ is a constant and that

$$
q>p>2
$$

For reasons that will appear later, we restrict ourselves to a class of planar domains $\Omega$ which satisfy certain geometric properties. We assume that, for some $L_{1}, L_{2}>0$,

$\Omega \subset \mathbb{R}^{2}$ is a smooth bounded domain of class $C^{2+\epsilon}$ for some $\epsilon \in(0,1)$;

$\Omega$ is symmetric with respect to the axis $x=0$;

$\Omega \subset\{y>0\}$ and $\Omega$ contains the rectangle $\left(-L_{1}, L_{1}\right) \times\left(0,2 L_{2}\right)$;

$\Omega$ is convex in the $x$-direction.

In particular, by (1.5), $\partial \Omega$ has a flat part, centered at the origin $(0,0)$. Note that assumption (1.6) is equivalent to the fact that $\Omega \cap\left\{y=y_{0}\right\}$ is a line segment for each $y_{0}$.

The initial data $u_{0}$ is taken in $\mathcal{V}_{\mu}$, where

$$
\mathcal{V}_{\mu}:=\left\{u_{0} \in C^{1}(\bar{\Omega}), u_{0} \geq \mu y \text { in } \Omega, u_{0}=\mu y \text { on } \partial \Omega\right\} .
$$

We shall use the following notation throughout:

$$
\Omega_{+}:=\{(x, y) \in \Omega ; x>0\} .
$$

For $T>0$, set $Q_{T}=\Omega \times(0, T), S_{T}=\partial \Omega \times(0, T)$ and $\partial_{P} Q_{T}=S_{T} \cup(\bar{\Omega} \times\{0\})$ its parabolic boundary.

Problem (1.1) is well posed locally in time (see Section 2 for details), with blow-up alternative in $W^{1, \infty}$ norm. For brevity, when no confusion arises, the existence time of its maximal solution $u$ will be denoted by

$$
T:=T_{\max }\left(u_{0}\right) \leq \infty
$$

It is known (see [2, 15]) that global nonexistence, i.e. $T<\infty$, occurs for suitably large initial data (more generally, for problem (1.1) in an $n$-dimensional bounded domain with Dirichlet boundary conditions). Note that the condition $q>p$ is sharp, since the solutions are global and bounded in $W^{1, \infty}$ if $1<q \leq p$ (see [25, 15]). Since it follows 
easily from the maximum principle that $u$ itself remains uniformly bounded on $Q_{T}$, global nonexistence can only occur through gradient blow-up, namely

$$
\sup _{Q_{T}}|u|<\infty \quad \text { and } \quad \lim _{t \rightarrow T}\|\nabla u(\cdot, t)\|_{\infty}=\infty .
$$

This is different from the usual blow-up, in which the $L^{\infty}$ norm of the solution tends to infinity as $t \rightarrow T_{\text {max }}$, which occurs for equations with zero-order nonlinearities, such as $u_{t}=\Delta_{p} u+u^{q}$ (see [7, 8]). The study of $-L^{\infty}$ or gradient- blow-up singularities, in particular their location, time and spatial structure is very much of interest for the understanding of the physical problems modelled by such equation, as well as for the mathematical richness that they involve. The $L^{\infty}$ blow-up for the equation $u_{t}=\Delta_{p} u+u^{q}$ has been extensively studied, both in the case of linear $(p=2)$ and nonlinear $(p>2)$ diffusion; see respectively the monographs [20] and [21] and the numerous references therein.

As for equation (1.1) with $p=q=2$, it is known as the (deterministic version of the) Kardar-Parisi-Zhang (KPZ) equation, describing the profile of a growing interface in certain physical models (see [11]), where $u$ then represents the height of the interface profile. The case of $p=2$ and $q \geq 1$ is a more general model which was developed by Krug and Spohn, aiming at studying the effect of the nonlinear gradient term on the properties of solutions (see [12]). Our main interest in this paper is to study the effect of a quasilinear gradient diffusivity on the localization of the singularities.

For the case of linear diffusion $p=2$, various sufficient conditions for gradient blowup and global existence were provided and qualitative properties were investigated, such as: nature of the blow-up set, rate and profile of blow-up, maximum existence time and continuation after blow-up, boundedness of global solutions and convergence to a stationary state. We refer for these to the works [1, 10, 23, 24, 20, 17] and the references therein.

The case $p>2$ is far from being completely understood and fewer works deal with the nonlinear diffusion. The large time behavior of global solutions in bounded or unbounded domains has been studied in [16, 25, 15, 5, 4, 14. Concerning the asymptotic description of singularities, results on the gradient blow-up rate in one space dimension can be found in [3, 26, 27]. On the other hand, in any space dimension, it is known [2] that gradient blow-up can take place only on the boundary, i.e.

$$
G B U S\left(u_{0}\right) \subset \partial \Omega,
$$

where the gradient blow-up set is defined by

$$
G B U S\left(u_{0}\right)=\left\{x_{0} \in \bar{\Omega} ; \text { for any } \rho>0, \sup _{\left(\bar{\Omega} \cap B_{\rho}\left(x_{0}\right)\right) \times(T-\rho, T)}|\nabla u|=\infty\right\} .
$$

Moreover, the following upper bounds for the space profile of the singularity were obtained in [2]:

$$
|\nabla u| \leq C \delta^{-\frac{1}{q-p+1}} \text { and } u \leq C \delta^{\frac{q-p}{q-p+1}} \text { in } Q_{T}, \quad \text { where } \delta(x)=\operatorname{dist}(x, \partial \Omega),
$$

and they are sharp in one space dimension [3]. (Our nondegeneracy lemma 7.1 below indicates that they are also sharp in higher dimensions.) 
It is easy to see, by considering radially symmetric solutions with $\Omega$ being a ball, that $G B U S\left(u_{0}\right)$ can be the whole of $\partial \Omega$. A natural question is then:

Can one produce examples (in more than one space dimension) when $G B U S\left(u_{0}\right)$ is a proper subset of $\partial \Omega$, especially a single point ?

The goal of this article is to provide an affirmative answer to this question. Our main result is the following.

Theorem 1.1. Assume (1.2)-(1.6).

(i) For any $\rho \in\left(0, L_{1}\right)$, there exists $\mu_{0}=\mu_{0}(p, q, \Omega, \rho)>0$ such that, for any $\mu \in$ $\left(0, \mu_{0}\right]$, there exist initial data $u_{0}$ in $\mathcal{V}_{\mu} \cap C^{2}(\bar{\Omega})$ such that the corresponding solution $u$ of (1.1) enjoys the following properties:

$$
T:=T_{\max }\left(u_{0}\right)<\infty \text { and } G B U S\left(u_{0}\right) \subset[-\rho, \rho] \times\{0\},
$$

$u(\cdot, t)$ is symmetric with respect to the line $x=0$, for all $t \in(0, T)$,

$$
\begin{gathered}
u_{x} \leq 0 \quad \text { in } \Omega_{+} \times(0, T), \\
u_{y} \geq \mu / 2 \quad \text { in } Q_{T} .
\end{gathered}
$$

(ii) For any such $\mu$ and $u_{0}$, we have

$$
\operatorname{GBUS}\left(u_{0}\right)=\{(0,0)\}
$$

A class of initial data satisfying the requirements of Theorem 1.1 is provided in Lemma 6.1 below. We note that, in the semilinear case $p=2$, a single-point boundary gradient blow-up result was obtained in [17]. Although we follow the same basic strategy, the proof here is considerably more complicated. We point out right away that, in view of property (1.11), the equation is not degenerate for the solutions under consideration. However, since the essential goal of this article is to study the effect of nonlinear diffusion on gradient blow-up, what is relevant here are the large values of the gradient in the diffusion operator (rather than the issues of loss of higher regularity that would arise from the degenerate nature of the equation near the level $\nabla u=0$ ). It is an open question whether or not single-point gradient blow-up can still be proved in the case $\mu=0$. Actually, the lower bound (1.11) on $|\nabla u|$ is crucially used at various points of the proof, which is already very long and involved, due to the presence of the nonlinear - even though nondegenerate - diffusion term (see the next subsection for more details).

Section 2 is devoted to local in time well-posedness and regularity results. The proof of Theorem 1.1 will be split into several sections, namely sections 3-7 for assertion (i) and sections 8-10 for assertion (ii) (the latter uses also section 5). Finally, in two appendices, we provide the proofs of some regularity properties and a suitable parabolic version of Serrin's corner lemma. Since the proof is quite long and involved, for the convenience of readers, we now give an outline of the main steps of the proof. 


\subsection{Outline of proof}

For sake of clarity, we have divided the proof into a number of intermediate steps, each of which being relatively short (one or two pages, say), except for step (f), which involves long and hard computations. For the convenience of readers, we outline the structure of the proof.

(a) Preliminary estimates (Lemmas 3.1 3.4): The symmetry in the variable $x$ and the decreasing property for $x>0$ are basic features in order to expect single-point gradient blow-up. Besides $u$ being bounded, we also have boundedness of $u_{t}$. Moreover, for sufficiently small $\mu$ and under a suitable assumption on $u_{0}$, we show that $u_{y} \geq \mu / 2$. These bounds on $u_{t}$ and $u_{y}$ seem necessary in the very long calculations of the key step (f) below. In turn, the positivity of $u_{y}$ guarantees that solutions are actually classical and that $D^{2} u, D^{3} u$ satisfy some bounds which seem also necessary to the argument, especially in view of the application of the Hopf lemma and the Serrin corner lemma in step (g).

(b) Finite time gradient blow-up for suitably concentrated initial data (Lemma 4.1): by using a rescaling argument and known blow-up criteria, we show that the solution blows up in finite time provided the initial data is suitably concentrated in a small ball near the origin.

(c) Local boundary gradient control (Lemma 5.1): if the gradient remains bounded on the boundary near a given boundary point, then the gradient remains also bounded near that point inside the domain, hence it is not a blow-up point. This is proved by a local Bernstein type argument.

(d) Localization of the gradient blow-up set (Lemma 5.3): if an initial data is suitably concentrated near the origin, then the gradient blow-up set is contained in a small neighborhood of the origin. This is proved by constructing comparison functions which provide a control of the gradient on the boundary outside a small neighborhood of the origin, and then applying step (c). One then constructs (Lemma 6.1) initial data which also fulfill the assumptions in (a) and (b). This ensures the existence of "well-prepared" initial data and thereby completes the proof of assertion (i) of Theorem 1.1.

(e) Nondegeneracy of gradient blow-up (Lemma 7.1): if the solution is only "weakly singular" in a neighborhood of a boundary point, then the singularity is removable. 1 More precisely, we show the existence of $m=m(p, q) \in(0,1)$ such that, for a given point $\left(x_{0}, 0\right)$ on the flat part of $\partial \Omega$, if $u(x, y, t) \leq c(x) y^{m}$ near $\left(x_{0}, 0\right)$ for $t$ close to $T$, then $\left(x_{0}, 0\right)$ is not a gradient blow-up point. In view of step (c), it suffices to control the gradient on the boundary near the point $\left(x_{0}, 0\right)$. This is achieved by constructing special comparison functions, taking the form of "regularizing (in time) barriers".

(f) Verification of a suitable parabolic inequality for an auxiliary function $J$, of the form

$$
J(x, y, t)=u_{x}+k x y^{-\gamma} u^{\alpha} .
$$

(Proposition 8.1). This is the most technical step and gives rise to very long computations. Those computations make use, among many other things, of the singular, Bernstein-type

\footnotetext{
${ }^{1}$ In this context, the term "nondegeneracy" describes a property of finite-time singularities (like in, e.g., Giga and Kohn [9]) and should not be confused with the notion of nondegenerate diffusion mentioned after Theorem 1.1.
} 
boundary gradient estimate (1.7), obtained in [2]. They use the bound on $u_{t}$ and the lower bound on $u_{y}$, obtained at step (a) (it is not clear if the latter could be relaxed 2 ).

We note that a similar function $J$ was introduced in [17] to treat the semilinear case $p=2$. The function in [17] was a 2D-modification of a one-dimensional device from [6], used there to show single point $L^{\infty}$ blow-up for radial solutions of equations of the form $u_{t}-\Delta_{p} u=u^{q}$ (for $p=2$, see also [8] for $p>2$ ). Although the ideas are related, the calculations here are considerably harder than in [6, 8, 17].

(g) Verification of initial-boundary conditions for the auxiliary function $J$ in a small subrectangle near the origin. This requires a delicate parabolic version of Serrin's corner lemma, which we prove in Appendix 2 (see Proposition 11.1).

(h) Derivation of a weakly singular gradient estimate near the origin and conclusion. Steps (d) and (e) imply $J \leq 0$ by the maximum principle. By integrating this inequality we obtain an inequality of the form $u(x, y, t) \leq c(x) y^{k}$ as $y \rightarrow 0$, for each small $x \neq 0$ and some $k>m$. In view of step (e), this shows that $(0,0)$ is the only gradient blow-up point.

\section{Local well-posedness and regularity}

In this section we consider the question of local existence and regularity for problem (1.1). Actually, we consider the slightly more general problem

$$
\begin{aligned}
u_{t}-\Delta_{p} u & =|\nabla v|^{q} \quad \text { in } Q_{T}, \\
u & =g \quad \text { on } S_{T}, \\
u(\cdot, 0) & =u_{0} \quad \text { in } \Omega,
\end{aligned}
$$

where the boundary data $g$ and initial data $u_{0}$ satisfy:

$g \geq 0$ is the trace on $\partial \Omega$ of a regular function in $C^{2+\gamma}(\bar{\Omega})$ for some $\gamma \in(0,1)$

and

$$
u_{0} \in W^{1, \infty}(\Omega), \quad u_{0} \geq 0, \quad u_{0}=g \quad \text { on } \partial \Omega .
$$

A function $u$ is called a weak super- (sub-) solution of problem (2.1)-(2.3) on $Q_{T}$ if $u(\cdot, 0) \geq(\leq) u_{0}$ in $\Omega, u \geq(\leq) g$ on $S_{T}$,

$$
u \in C(\bar{\Omega} \times[0, T)) \cap L^{q}\left(0, T ; W^{1, q}(\Omega)\right), \quad u_{t} \in L^{2}\left(0, T ; L^{2}(\Omega)\right)
$$

and the integral inequality

$$
\iint_{Q_{T}} u_{t} \psi+|\nabla u|^{p-2} \nabla u \cdot \nabla \psi d x d t \geq(\leq) \iint_{Q_{T}}|\nabla u|^{q} \psi d x d t
$$

holds for all $\psi \in C^{0}\left(\overline{Q_{T}}\right) \cap L^{p}\left(0, T ; W^{1, p}(\Omega)\right)$ such that $\psi \geq 0$ and $\psi=0$ on $S_{T}$. A function $u$ is a weak solution of (2.1)-(2.3) if it is a super-solution and a sub-solution.

The following result was established in [2, Theorem 1.1] (actually in any space dimension).

\footnotetext{
${ }^{2}$ It seems that the constants in some of the key estimates there are nonuniform as $\mu \rightarrow 0^{+}$, which prevents us to argue by a limiting procedure from the case $\mu>0$.
} 
Theorem 2.1. Assume (1.3) and $q>p-1>1$. Let $M_{1}>0$, let $u_{0}, g$ satisfy (2.4) -(2.5) and $\left\|\nabla u_{0}\right\|_{\infty} \leq M_{1}$. Then:

(i) There exists a time $T_{0}=T_{0}\left(p, q, \Omega, M_{1},\|g\|_{C^{2}}\right)>0$ and a weak solution $u$ of (2.1) (2.3) on $\left[0, T_{0}\right)$, which moreover satisfies $u \in L^{\infty}\left(0, T_{0} ; W^{1, \infty}(\Omega)\right)$. Furthermore, $\nabla u$ is locally Hölder continuous in $Q_{T_{0}}$.

(ii) For any $\tau>0$, the problem (2.1) -(2.3) has at most one weak solution $u$ such that $u \in L^{\infty}\left(0, \tau ; W^{1, \infty}(\Omega)\right)$.

(iii) There exists a (unique) maximal, weak solution of (2.1)-(2.3) in $L_{l o c}^{\infty}\left([0, T) ; W^{1, \infty}(\Omega)\right)$, still denoted by $u$, with existence time denoted by $T=T_{\max }\left(u_{0}\right)$. Then

$$
\min _{\bar{\Omega}} u_{0} \leq u \leq \max _{\bar{\Omega}} u_{0} \quad \text { in } Q_{T},
$$

$\nabla u$ is locally Hölder continuous in $Q_{T}$ and

$$
\text { if } T<\infty \text {, then } \lim _{t \rightarrow T}\|\nabla u(t)\|_{\infty}=\infty \text { (gradient blow up, GBU). }
$$

Remark 2.1. We also have a comparison principle for problem (2.1)-(2.3), cf. [2, Proposition 2.1]. More precisely, if $v_{1}, v_{2} \in C\left(\bar{Q}_{T}\right)$ are weak sub-/super-solutions of (2.1) -(2.3) in $Q_{T}$, then

$$
\sup _{Q_{T}}\left(v_{1}-v_{2}\right) \leq \sup _{\partial Q_{T}}\left(v_{1}-v_{2}\right)
$$

As one expects, the solution will possess additional regularity if we know that $|\nabla u|$ remains bounded away from 0 . This is made precise by the following result, which is a consequence of regularity theory [13, 18] for quasilinear uniformly parabolic equations. However, for completeness, we provide a proof in Appendix 1.

Theorem 2.2. Under the assumptions of Theorem[2.1, suppose also that $\inf _{Q_{T}}|\nabla u|>0$.

(i) Then $u$ is a classical solution in $Q_{T}$ and

$$
u \in C_{l o c}^{2+\alpha, 1+\alpha / 2}(\bar{\Omega} \times(0, T)) .
$$

for some $\alpha \in(0,1)$.

(ii) Moreover,

$$
\nabla u \in C_{l o c}^{2+\beta, 1+\beta / 2}(\Omega \times(0, T)) .
$$

for some $\beta \in(0,1)$.

(iii) If the boundary conditions in (2.2) depend only on $y$, then

$$
u_{x} \in C_{l o c}^{2+\beta, 1+\beta / 2}(\bar{\Omega} \times(0, T)) .
$$

for some $\beta \in(0,1)$. 


\section{Preliminary estimates: $x$-symmetry, lower bound on $u_{y}$ and bound on $u_{t}$}

Notation. Throughout the paper, we shall use the summation convention on repeated indices, in expressions of the form $a_{i j} u_{i j}$ or $a_{i j} u_{i} u_{j}$. Also, the letter $C$ will denote positive constants which may vary from line to line, and whose dependence will be indicated if necessary.

Lemma 3.1. Let $\mu>0$ and $u_{0} \in \mathcal{V}_{\mu}$. Assume

$$
\begin{aligned}
& u_{0} \text { is symmetric with respect to the line } x=0, \\
& \qquad \partial_{x} u_{0} \leq 0 \text { in } \Omega_{+} .
\end{aligned}
$$

Then we have

$$
u(x, y, t) \geq \mu y \quad \text { in } Q_{T} .
$$

and properties (1.9)-(1.10) are satisfied.

Proof. Property (1.9) is a direct consequence of (3.1) and the local-in-time uniqueness. Due to the assumption $u_{0} \in \mathcal{V}_{\mu}, \underline{v}=\mu y$ is a subsolution of (1.1). This implies (3.3).

To prove (1.10), fix $h>0$ and let

$$
u_{ \pm}=u(x \pm h, y, t) \quad \text { for }(x, y) \in \Omega_{h}:=\left\{(x, y) \in \Omega_{+} ;(x+h, y) \in \Omega\right\} \text { and } t \in(0, T) .
$$

Owing to (1.4) and (1.6), we see that $(x-h, y) \in \Omega$ for all $(x, y) \in \Omega_{h}$, so that $u_{-}$is well defined. The functions $u_{ \pm}$are weak solutions of (1.1) $)_{1}$ in $\Omega_{h} \times(0, T)$. Also $u_{+} \leq u_{-}$at $t=0$, due to (3.1)-(3.2) and (1.6).

Let $(x, y) \in \partial \Omega_{h}$. If $x=0$, then $u_{+}(x, y, t)=u(h, y, t)=u(-h, y, t)=u_{-}(x, y, t)$ by (1.9). If $x>0$, then $(x+h, y) \in \partial \Omega$ as a consequence of $(x, y) \in \partial \Omega_{h}$ and (1.6). So, by (3.3), we have

$$
u_{+}(x, y, t)=u(x+h, y, t)=\mu y \leq u_{-}(x-h, y, t) .
$$

We deduce from the comparison principle that $u_{+} \leq u_{-}$in $\Omega_{h} \times(0, T)$, which implies (1.10).

Our next lemma provides a useful supersolution of problem (1.1).

Lemma 3.2. For $0<\rho<L_{1}$, denote $\Sigma_{\rho}=[-\rho / 2, \rho / 2] \times\{0\}$ and $\Sigma_{\rho}^{\prime}=\partial \Omega \backslash((-\rho, \rho) \times$ $\{0\})$. There exist $\mu_{0}=\mu_{0}(p, q, \Omega, \rho)>0$ and a function $U \in C^{2}(\bar{\Omega})$, depending on $p, q, \rho$, with the following properties:

$$
\begin{aligned}
& U>0 \quad \text { on } \Omega \cup \Sigma_{\rho}, \\
& U=0 \quad \text { on } \Sigma_{\rho}^{\prime}, \\
& \left|U_{y}\right| \leq 1 / 2 \quad \text { in } \Omega
\end{aligned}
$$

and, for all $0<\mu \leq \mu_{0}$, the function $\bar{U}=\mu(y+U)$ satisfies

$$
-\Delta_{p} \bar{U} \geq|\nabla \bar{U}|^{q} \quad \text { in } \Omega
$$


Proof. Fix a nonnegative function $\phi \in C^{3}\left(\mathbb{R}^{2}\right)$ such that $\phi=1$ on $\Sigma_{\rho}$ and $\phi=0$ on $\Sigma_{\rho}^{\prime}$. We shall look for $U$ under the form $U=\varepsilon V, \varepsilon>0$, where $V \in C^{2}(\bar{\Omega})$ is the classical solution of the linear elliptic problem

$$
\begin{cases}-\left[V_{x x}+(p-1) V_{y y}\right]=1 & \text { in } \Omega, \\ V=\phi & \text { on } \partial \Omega .\end{cases}
$$

Note that $V>0$ in $\Omega$ by the strong maximum principle which, along with the boundary conditions in (3.8), will guarantee (3.4) $-(3.5)$. Let $\bar{U}=\mu(y+U)$. Assume $0<\varepsilon<$ $1 /\left(\left\|V_{x}\right\|_{\infty}+2\left\|V_{y}\right\|_{\infty}\right)$, which implies (3.6), as well as $\left|1+\varepsilon V_{y}\right| \geq 1 / 2$ and $|\nabla \bar{U}| \leq 2 \mu$. To check (3.7), we compute:

$$
\begin{aligned}
\Delta_{p} \bar{U} & =|\nabla \bar{U}|^{p-2}\left[\Delta \bar{U}+(p-2) \frac{\bar{U}_{i} \bar{U}_{j} \bar{U}_{i j}}{|\nabla \bar{U}|^{2}}\right] \\
& =\mu \varepsilon|\nabla \bar{U}|^{p-2}\left[V_{x x}+V_{y y}+(p-2) \frac{\left(\varepsilon V_{x}\right)^{2} V_{x x}+2\left(\varepsilon V_{x}\right)\left(1+\varepsilon V_{y}\right) V_{x y}+\left(1+\varepsilon V_{y}\right)^{2} V_{y y}}{\left(1+\varepsilon V_{y}\right)^{2}+\left(\varepsilon V_{x}\right)^{2}}\right] \\
& =\mu \varepsilon|\nabla \bar{U}|^{p-2}\left[V_{x x}+(p-1) V_{y y}+(p-2) \frac{\left(\varepsilon V_{x}\right)^{2}\left(V_{x x}-V_{y y}\right)+2\left(\varepsilon V_{x}\right)\left(1+\varepsilon V_{y}\right) V_{x y}}{\left(1+\varepsilon V_{y}\right)^{2}+\left(\varepsilon V_{x}\right)^{2}}\right] \\
& \leq \mu \varepsilon|\nabla \bar{U}|^{p-2}\left[-1+C \varepsilon^{2}+C \varepsilon\right]
\end{aligned}
$$

in $\Omega$, with $C>0$ depending only on $\Omega, \rho, p$ (through $V$ ). We may then choose $\varepsilon$ depending only on $\Omega, \rho, p$, such that $-\Delta_{p} \bar{U} \geq \frac{\mu \varepsilon}{2}|\nabla \bar{U}|^{p-2}$. Next, since $q>p$, for any $\mu \leq \mu_{0}$ with $\mu_{0}=\mu_{0}(p, q, \Omega, \rho)>0$ sufficiently small, we have

$$
-\Delta_{p} \bar{U} \geq \frac{\mu \varepsilon}{2}|\nabla \bar{U}|^{p-2} \geq \frac{\mu \varepsilon}{2(2 \mu)^{q+2-p}}|\nabla \bar{U}|^{q} \geq|\nabla \bar{U}|^{q} \quad \text { in } \Omega .
$$

Based on Lemma 3.2, we construct a class of solutions such that $u_{y}$ satisfies a positive lower bound.

Lemma 3.3. Let $0<\rho<L_{1}$ and let $\mu_{0}, \bar{U}$ be given by Lemma 3.2. Assume that $0<\mu \leq \mu_{0}$ and $u_{0} \in \mathcal{V}_{\mu}$ satisfy

$$
u_{0}(x, y) \leq \mu\left(y+c \chi_{(-\rho / 2, \rho / 2) \times\left(0, L_{2}\right)}\right) \quad \text { in } \Omega,
$$

with $c=c(p, q, \Omega, \rho)>0$ sufficiently small.

(i) Then $u \leq \bar{U}$ in $Q_{T}$.

(ii) Assume in addition that

$$
\partial_{y} u_{0} \geq \mu / 2 \quad \text { in } \Omega
$$

Then

$$
\partial_{y} u \geq \mu / 2 \quad \text { in } Q_{T}
$$


Proof. (i) Let $\bar{U}=\mu(y+U)$ be given by Lemma 3.2, From (3.4), we know that

$$
c:=\min _{[-\rho / 2, \rho / 2] \times\left[0, L_{2}\right]} U>0 .
$$

Under assumption (3.9), we thus have $u_{0} \leq \bar{U}$ in $\Omega$. Since $u=\mu y \leq \bar{U}$ on $S_{T}$, we infer from the comparison principle that $u \leq \bar{U}$ in $Q_{T}$.

(ii) Set $\delta_{0}=\mu / 2$, fix $h>0$ and let $\tilde{\Omega}_{h}=\{(x, y) \in \Omega ;(x, y+h) \in \Omega\}$. We observe that

$$
u_{1}:=u(x, y, t)+\delta_{0} h \quad \text { and } \quad \mathrm{u}_{2}:=u(x, y+h, t)
$$

are weak solutions of $(1.1)_{1}$ in $\tilde{\Omega}_{h} \times(0, T)$.

Let $(x, y) \in \partial \tilde{\Omega}_{h}$. If $(x, y) \in \partial \Omega$, then, by (3.3), we have

$$
u_{2}(x, y, t)=u(x, y+h, t) \geq \mu(y+h)=u(x, y, t)+\mu h \geq u_{1}(x, y, t) .
$$

Otherwise, we have $(x, y) \in \Omega$ and $(x, y+h) \in \partial \Omega$. So there is a minimal $\tilde{h} \in(0, h]$ such that $(x, y+\tilde{h}) \in \partial \Omega$. By the mean-value inequality, it follows that, for some $\theta \in(0,1)$,

$$
U(x, y)=U(x, y+\tilde{h})-\tilde{h} U_{y}(x, y+\theta \tilde{h}) \leq\left|U_{y}(x, y+\theta \tilde{h})\right| h \leq h / 2,
$$

where we used (3.6). Therefore, $\bar{U}(x, y) \leq \mu(y+h / 2)$, hence

$$
u(x, y+h, t)-u(x, y, t) \geq \mu(y+h)-\bar{U}(x, y) \geq \frac{\mu h}{2} .
$$

We have thus proved that

$$
u_{2} \geq u_{1} \text { on } \partial \tilde{\Omega}_{h} .
$$

On the other hand, using (3.10) and the fact that $u_{0}=\mu y$ on $\partial \Omega$, it is not difficult to show that $y \mapsto u_{0}(x, y)-\delta_{0} y$ is nondecreasing in $\Omega$. (Note that in case $\Omega$ is nonconvex, this is not a mere consequence of (3.10) alone). It follows that

$$
u(x, y+h, 0) \geq u(x, y, 0)+\delta_{0} h \quad \text { in } \tilde{\Omega}_{h} .
$$

Owing to (3.12)-(3.13), we may then apply the comparison principle to deduce that $u_{2} \geq$ $u_{1}$ in $\tilde{\Omega}_{h} \times(0, T)$. Since $h$ is arbitrary, the desired conclusion (3.11) follows immediately.

Assuming that $u_{0}$ is sufficiently regular, we also get an estimate on the time derivative.

Lemma 3.4. Let $\mu \geq 0$ and assume that $u_{0} \in \mathcal{V}_{\mu} \cap C^{2}(\bar{\Omega})$. Then

$$
\left|u_{t}\right| \leq \tilde{C}_{1}:=\left\|\Delta_{p} u_{0}+\left|\nabla u_{0}\right|^{q}\right\|_{\infty} \quad \text { in } Q_{T} .
$$

Proof. It is easy to see that $v_{ \pm}(x, y, t):=u_{0}(x, y) \pm \tilde{C}_{1} t$ are respectively super- and sub-solution of (1.1) in $Q_{T}$. The comparison principle implies that

$$
u_{0}(x, y)-\tilde{C}_{1} t \leq u(x, y, t) \leq u_{0}(x, y)+\tilde{C}_{1} t \quad \text { in } Q_{T} .
$$

Now fix $h \in(0, T)$ and set $w_{ \pm}(x, y, t):=u(x, y, t+h) \pm \tilde{C}_{1} h$. By (3.15), we have $w_{-}(x, y, 0) \leq u_{0}(x, y) \leq w_{+}(x, y, 0)$ and it follows that $w_{ \pm}$are respectively super- and sub-solution of (1.1) in $Q_{T-h}$. By a further application of the comparison principle, we deduce that

$$
|u(x, y, t+h)-u(x, y, t)| \leq \tilde{C}_{1} h \quad \text { in } Q_{T-h} .
$$

Since $h$ is arbitrary, we conclude by dividing by $h$ and sending $h \rightarrow 0$. 


\section{Finite-time gradient blow-up for concentrated ini- tial data}

In this section, by a rescaling argument, we show that the solution of (1.1) blows up in finite time provided the initial data is suitably concentrated in a small ball near the origin. For such concentrated initial data, under some additional assumptions, we will show in section 6 that the gradient blow-up set is contained in a small neighborhood of the origin.

The following lemma shows in particular that gradient blow-up may occur for initial data of arbitrarily small $L^{\infty}$-norm (but the $W^{1, \infty}$ norm has to be sufficiently large). We note that we do not assume (3.10) here, so that in the proof, we work only with weak solutions (in particular, we cannot use the continuity of $\nabla u$ up to the boundary).

Lemma 4.1. Let $\kappa=(q-p) /(q-p+1)$. There exists $C_{1}=C_{1}(p, q)>0$ such that, if $\varepsilon \in\left(0, \min \left(L_{1}, L_{2}\right)\right), \mu \geq 0$ and $u_{0} \in \mathcal{V}_{\mu}$ satisfies

$$
u_{0}(x, y) \geq C_{1} \varepsilon^{\kappa} \quad \text { in } B_{\varepsilon / 3}(0, \varepsilon) \subset \Omega,
$$

then $T_{\max }\left(u_{0}\right)<\infty$.

Proof. We denote $B_{r}=B_{r}(0,0) \subset \mathbb{R}^{2}$ for $r>0$. Fix a radially symmetric function $h \in C_{0}^{\infty}\left(B_{1}\right), h \geq 0$, such that $\operatorname{supp}(h) \subset B_{1 / 3}$ and $\|h\|_{\infty}=1$. We consider the following problem

$$
\begin{cases}v_{t}-\Delta_{p} v=|\nabla v|^{q}, & x \in B_{1}, \quad t>0 \\ v(x, y, t)=0, & x \in \partial B_{1}, \quad t>0 \\ v(x, y, 0)=v_{0}(x, y):=C_{1} h(x, y), & x \in B_{1} .\end{cases}
$$

We know from [2, Theorem 1.4] that there exists $C_{0}=C_{0}(p, q)>0$ such that if $\left\|v_{0}\right\|_{L^{1}} \geq$ $C_{0}$, then $T_{\max }\left(v_{0}\right)<\infty$. Therefore, if we take $C_{1}=C_{1}(p, q)>0$ large enough then $\nabla v$ blows up in finite time in $L^{\infty}$ norm.

Next we use the scale invariance of the equation, considering the rescaled functions

$$
v_{\varepsilon}(x, y, t):=\varepsilon^{\kappa} v\left(\frac{x}{\varepsilon}, \frac{y-\varepsilon}{\varepsilon}, \frac{t}{\varepsilon^{(2 q-p) /(q-p+1)}}\right) .
$$

Pick $\varepsilon \in\left(0, \min \left(L_{1}, L_{2}\right)\right)$ and denote $\tilde{B}_{\varepsilon}=B_{\varepsilon}(0, \varepsilon)$, which is included in $\Omega$ and tangent to $\partial \Omega$ at the origin. Set $T_{\varepsilon}=\varepsilon^{(2 q-p) /(q-p+1)} T_{\max }\left(v_{0}\right)$ and $\tilde{T}_{\varepsilon}=\min \left(T_{\max }\left(u_{0}\right), T_{\varepsilon}\right)$. We shall show that, for each $\tau \in\left(0, \tilde{T}_{\varepsilon}\right)$,

$$
\left\|\nabla v_{\varepsilon}\right\|_{L^{\infty}\left(Q_{\tau}\right)} \leq \max \left(\left\|\nabla v_{\varepsilon}(\cdot, 0)\right\|_{\infty},\|\nabla u\|_{L^{\infty}\left(Q_{\tau}\right)}\right) .
$$

Since gradient blow-up occurs in finite time $T_{\varepsilon}$ for $v_{\varepsilon}$, this will guarantee $T_{\max }\left(u_{0}\right) \leq T_{\varepsilon}<$ $\infty$.

First observe that $v_{\varepsilon}$ solves (4.2) in $\tilde{B}_{\varepsilon} \times\left(0, T_{\varepsilon}\right)$, with initial data $v_{\varepsilon}(x, y, 0) \leq u_{0}(x, y)$, due to (4.1). It follows from the comparison principle that $v_{\varepsilon} \leq u$ in $\tilde{B}_{\varepsilon} \times\left(0, \tilde{T}_{\varepsilon}\right)$. In particular, for each $h \in(0, \varepsilon)$ and $t \in\left(0, \tilde{T}_{\varepsilon}\right)$, since $u(0,0, t)=0$, we get that

$$
\frac{v_{\varepsilon}(0, h, t)}{h} \leq \frac{u(0, h, t)}{h} \leq\|\nabla u(\cdot, t)\|_{\infty}
$$


We shall next show that the first quantity in (4.4) can be suitably bounded from below in terms of the sup norm of $\nabla v_{\varepsilon}$.

Fix $h \in(0, \varepsilon)$ and let $\tilde{B}_{\varepsilon}^{h}:=B_{\varepsilon}(0, \varepsilon-h)$ Since $v_{\varepsilon}^{h}(x, y, t):=v_{\varepsilon}(x, y+h, t)$ is a solution of (4.2) in $\tilde{B}_{\varepsilon}^{h}$, it follows from the comparison principle (see Remark 2.1) that, for any $0<\tau<\tilde{T}_{\varepsilon}$,

$$
\begin{aligned}
& \sup _{\left(\tilde{B}_{\varepsilon} \cap \tilde{B}_{\varepsilon}^{h}\right) \times(0, \tau)}\left|v_{\varepsilon}(x, y+h, t)-v_{\varepsilon}(x, y, t)\right| \\
& \leq \max \left(\sup _{\tilde{B}_{\varepsilon} \cap \tilde{B}_{\varepsilon}^{h}}\left|v_{\varepsilon}^{h}(x, y, 0)-v_{\varepsilon}(x, y, 0)\right|, \sup _{\partial\left(\tilde{B}_{\varepsilon} \cap \tilde{B}_{\varepsilon}^{h}\right) \times(0, \tau)}\left|v_{\varepsilon}^{h}(x, y, t)-v_{\varepsilon}(x, y, t)\right|\right) .
\end{aligned}
$$

We claim that, for any $0<t<T_{\varepsilon}$,

$$
\sup _{\partial\left(\tilde{B}_{\varepsilon} \cap \tilde{B}_{\varepsilon}^{h}\right)}\left|v_{\varepsilon}^{h}(x, y, t)-v_{\varepsilon}(x, y, t)\right| \leq v_{\varepsilon}(0, h, t) .
$$

First consider the case $(x, y) \in \partial \tilde{B}_{\varepsilon}$. Then $\|(x, y+h)-(0, \varepsilon)\| \geq\|(0, h)-(0, \varepsilon)\|$, due to

$$
x^{2}+(y+h-\varepsilon)^{2}-(h-\varepsilon)^{2}=\varepsilon^{2}-(y-\varepsilon)^{2}+(y+h-\varepsilon)^{2}-(h-\varepsilon)^{2}=2 h y \geq 0 .
$$

Since $v_{\varepsilon}$ is radially symmetric and non-increasing with respect to the point $(0, \varepsilon)$, we deduce that

$$
\left|v_{\varepsilon}(x, y+h, t)-v_{\varepsilon}(x, y, t)\right|=v_{\varepsilon}(x, y+h, t) \leq v_{\varepsilon}(0, h, t) .
$$

Next consider the case $(x, y) \in \partial \tilde{B}_{\varepsilon}^{h}$ that is, $(x, y+h) \in \partial \tilde{B}_{\varepsilon}$. Then $\|(x, y)-(0, \varepsilon)\| \geq$ $\|(0, h)-(0, \varepsilon)\|$, due to

$$
x^{2}+(y-\varepsilon)^{2}-(h-\varepsilon)^{2}=\varepsilon^{2}-(y+h-\varepsilon)^{2}+(y-\varepsilon)^{2}-(h-\varepsilon)^{2}=2 h(2 \varepsilon-h-y) \geq 0 .
$$

Therefore, $\left|v_{\varepsilon}(x, y+h, t)-v_{\varepsilon}(x, y, t)\right|=v_{\varepsilon}(x, y, t) \leq v_{\varepsilon}(0, h, t)$, and the claim (4.6) is proved.

Now fix $y \in[0, \varepsilon)$. It follows from (4.4)-(4.6) that, for each $0<h<\varepsilon-y$ and $0<t<\tau<\tilde{T}_{\varepsilon}$

$$
\frac{\left|v_{\varepsilon}(0, y+h, t)-v_{\varepsilon}(0, y, t)\right|}{h} \leq \max \left(\left\|\nabla v_{\varepsilon}(\cdot, 0)\right\|_{\infty},\|\nabla u\|_{L^{\infty}\left(Q_{\tau}\right)}\right)
$$

hence, letting $h \rightarrow 0$,

$$
\left|\partial_{y} v_{\varepsilon}(0, y, t)\right| \leq \max \left(\left\|\nabla v_{\varepsilon}(\cdot, 0)\right\|_{\infty},\|\nabla u\|_{L^{\infty}\left(Q_{\tau}\right)}\right) .
$$

For each $0<\tau<\tilde{T}_{\varepsilon}$, taking supremum over $y \in[0, \varepsilon)$ and $t \in(0, \tau)$ and using the fact that $v_{\varepsilon}$ is radially symmetric, we obtain (4.3). This concludes the proof of the lemma. 


\section{Local boundary control for the gradient and local- ization of the gradient blow-up set}

For simplicity we shall here assume (3.9) and (3.10), so as to have the continuity of $\nabla u$ up to the boundary (although one might possibly relax this assumption at the expense of additional work).

Lemma 5.1. Let $\rho, \mu, u_{0}$ be as in Lemma 3.3(ii) and let $\left(x_{0}, y_{0}\right) \in \partial \Omega$. If there exist $M_{0}, R>0$ such that

$$
|\nabla u| \leq M_{0} \quad \text { in }\left(B_{R}\left(x_{0}, y_{0}\right) \cap \partial \Omega\right) \times\left[0, T_{\max }\left(u_{0}\right)\right),
$$

then $\left(x_{0}, y_{0}\right)$ is not a gradient blow-up point.

The proof is based on a local Bernstein technique. For $\left(x_{0}, y_{0}\right) \in \partial \Omega, R>0$ and given $\alpha \in(0,1)$, we may select a cut-off function $\eta \in C^{2}\left(\bar{B}_{R}\left(x_{0}, y_{0}\right)\right)$, with $0<\eta \leq 1$, such that

$$
\eta=1 \text { on } \bar{B}_{R / 2}\left(x_{0}, y_{0}\right), \quad \eta=0 \text { on } \partial B_{R}\left(x_{0}, y_{0}\right)
$$

and

$$
\left.\begin{array}{rl}
|\nabla \eta| & \leq C R^{-1} \eta^{\alpha} \\
\left|D^{2} \eta\right|+\eta^{-1}|\nabla \eta|^{2} & \leq C R^{-2} \eta^{\alpha}
\end{array}\right\} \quad \text { on } \bar{B}_{R}\left(x_{0}, y_{0}\right)
$$

where $C=C(\alpha)>0$ (see e.g. [24] for an example of such function). Also, for $0<t_{0}<$ $\tau<T=T_{\max }\left(u_{0}\right)$, we denote

$$
Q_{\tau, R}^{t_{0}}=\left(B_{R}\left(x_{0}, y_{0}\right) \cap \Omega\right) \times\left(t_{0}, \tau\right) .
$$

For the proof of Lemma 5.1, we then rely on the following lemma from [2] (cf. [2, Lemma 3.1]; it was used there to derive upper estimates on $|\nabla u|$ away from the boundary).

Lemma 5.2. Let $\mu \geq 0$ and $u_{0} \in \mathcal{V}_{\mu}$. Let $\left(x_{0}, y_{0}\right) \in \partial \Omega, R>0,0<t_{0}<\tau<T$ and choose $\alpha=(q+1) /(2 q-p+2)$. Denote $w=|\nabla u|^{2}$ and $z=\eta w$. Then $z \in C^{2,1}\left(Q_{\tau, R}^{t_{0}}\right)$ and satisfies the following differential inequality

$$
\mathcal{L} z+C_{2} z^{\frac{2 q-p+2}{2}} \leq C_{3}\left(\frac{\left\|u_{0}\right\|_{\infty}}{t_{0}}\right)^{\frac{2 q-p+2}{q}}+C_{3} R^{-\frac{2 q-p+2}{q-p+1}}
$$

where $C_{i}=C_{i}(p, q)>0$,

$$
\begin{gathered}
\mathcal{L} z=z_{t}-\overline{\mathcal{A}} z-\bar{H} \cdot \nabla z \\
\overline{\mathcal{A}} z=|\nabla u|^{p-2} \Delta z+(p-2)|\nabla u|^{p-4}(\nabla u)^{t} D^{2} z \nabla u,
\end{gathered}
$$

and $\bar{H}$ is defined by

$$
\begin{aligned}
\bar{H}:= & {\left[(p-2) w^{\frac{p-4}{2}} \Delta u+\frac{(p-2)(p-4)}{2} w^{\frac{p-6}{2}} \nabla u \cdot \nabla w+q w^{\frac{q-2}{2}}\right] \nabla u } \\
& +\frac{p-2}{2} w^{\frac{p-4}{2}} \nabla w .
\end{aligned}
$$


Proof of Lemma 5.1. Let $t_{0}=T / 2<\tau<T$ and set

$$
M_{1}:=\sup _{0 \leq t \leq t_{0}}\|\nabla u\|_{L^{\infty}}<\infty
$$

By Lemma 3.3(ii) and Theorem 2.2, we know that $\nabla u$ is a continuous function on $\bar{\Omega} \times$ $(0, T)$, hence $z \in C\left(\overline{Q_{\tau, R}^{t_{0}}}\right)$. Therefore, unless $z \equiv 0$ in $\overline{Q_{\tau, R}^{t_{0}}}, z$ must reach a positive maximum at some point $\left(x_{1}, y_{1}, t_{1}\right) \in \overline{Q_{\tau, R}^{t_{0}}}$. Since

$$
z=0 \quad \text { on }\left(\partial B_{R}\left(x_{0}, y_{0}\right) \cap \bar{\Omega}\right) \times\left[t_{0}, \tau\right],
$$

we deduce that either $\left(x_{1}, y_{1}\right) \in B_{R}\left(x_{0}, y_{0}\right) \cap \Omega$ or $\left(x_{1}, y_{1}\right) \in B_{R}\left(x_{0}, y_{0}\right) \cap \partial \Omega$.

- If $t_{1}=t_{0}$, then

$$
z\left(x_{1}, y_{1}, t_{1}\right) \leq\left\|\nabla u\left(t_{0}\right)\right\|_{L^{\infty}}^{2} \leq M_{1}^{2} .
$$

- If $t_{0}<t_{1} \leq \tau$ and $\left(x_{1}, y_{1}\right) \in B_{R}\left(x_{0}, y_{0}\right) \cap \partial \Omega$, then, by (5.1),

$$
z\left(x_{1}, y_{1}, t_{1}\right) \leq M_{0}^{2} \text {. }
$$

- Next consider the case $t_{0}<t_{1} \leq \tau$ and $\left(x_{1}, y_{1}\right) \in B_{R}\left(x_{0}, y_{0}\right) \cap \Omega$. Then we have $\nabla z\left(x_{1}, y_{1}, t_{1}\right)=0, z_{t}\left(x_{1}, y_{1}, t_{1}\right) \geq 0$ and $D^{2} z\left(x_{1}, y_{1}, t_{1}\right) \leq 0$, and therefore $\mathcal{L} z \geq 0$. Using (5.3) we arrive at

$$
C_{2} z\left(x_{1}, y_{1}, t_{1}\right)^{\frac{2 q-p+2}{2}} \leq C_{3}\left(\frac{\left\|u_{0}\right\|_{\infty}}{t_{0}}\right)^{\frac{2 q-p+2}{q}}+C_{3} R^{-\frac{2 q-p+2}{q-p+1}}
$$

that is,

$$
\sqrt{z\left(x_{1}, y_{1}, t_{1}\right)} \leq C\left(\frac{\left\|u_{0}\right\|_{\infty}}{t_{0}}\right)^{\frac{1}{q}}+C R^{-\frac{1}{q-p+1}}=: M_{2}>0 .
$$

It follows from (5.7)-(5.10) that

$$
\frac{\max }{Q_{\tau, R}^{t_{0}}} z \leq M_{3}^{2}, \quad \text { with } M_{3}=\max \left\{M_{0}, M_{1}, M_{2}\right\}
$$

Since $z=|\nabla u|^{2}$ in $\left(B_{R / 2}\left(x_{0}, y_{0}\right) \cap \bar{\Omega}\right) \times\left(t_{0}, \tau\right)$ and $\tau \in\left(t_{0}, T\right)$ is arbitrary, we get

$$
|\nabla u| \leq M_{3} \quad \text { in }\left(B_{R / 2}\left(x_{0}, y_{0}\right) \cap \bar{\Omega}\right) \times\left(t_{0}, T\right),
$$

and we conclude that $\left(x_{0}, y_{0}\right)$ is not a gradient blow-up point.

By combining Lemmas 3.3 and 5.1, we can now easily obtain a class of initial data whose possible gradient blow-up set is contained in a small neighborhood of the origin.

Lemma 5.3. Let $\rho, \mu, u_{0}$ be as in Lemma 3.3(ii). Then $G B U S\left(u_{0}\right) \subset[-\rho, \rho] \times\{0\}$.

Proof. Denote again $\Sigma_{\rho}=[-\rho / 2, \rho / 2] \times\{0\}$ and $\Sigma_{\rho}^{\prime}=\partial \Omega \backslash([-\rho, \rho] \times\{0\})$. In view of Lemma 5.1, it suffices to show that

$$
\sup _{(x, y) \in \Sigma_{\rho}^{\prime}, t \in(0, T)}|\nabla u(x, y, t)|<\infty .
$$


But (5.11) easily follows from a comparison with the function $\bar{U}$ provided in Lemma 3.2 . Indeed, under the assumptions of Lemma 3.3(i), we already know that $u \leq \bar{U}$ in $Q_{T}$. Also, $u=\mu y=\bar{U}$ on $\Sigma_{\rho}^{\prime} \times(0, T)$. From this, along with (3.3), it follows that

$$
\partial_{\nu} \bar{U} \leq \partial_{\nu} u \leq \mu \partial_{\nu} y \text { on } \Sigma_{\rho}^{\prime} \times(0, T) .
$$

From (15.12) and $(1.1)_{2}$, we get

$$
|\nabla u|^{2} \leq \mu^{2}+\left|\partial_{\nu} u\right|^{2} \leq C \text { on } \Sigma_{\rho}^{\prime} \times(0, T),
$$

hence (5.11), and the lemma is proved.

\section{Existence of well-prepared initial data: proof of Theorem 1.1(i)}

We need to construct initial data meeting the requirements from sections $3-5$. This will be achieved in the following lemma. Let us fix an even function $\varphi \in C^{\infty}(\mathbb{R})$ such that $s \varphi^{\prime}(s) \leq 0$, with

$$
\varphi(s)= \begin{cases}1 & \text { for }|s| \leq 1 / 3 \\ 0 & \text { for }|s| \geq 2 / 3\end{cases}
$$

Lemma 6.1. Let $\kappa=(q-p) /(q-p+1)$ and let $C_{1}=C_{1}(p, q)>0$ be given by Lemma 4.1. For $\varepsilon \in\left(0, \min \left(L_{1}, L_{2} / 2\right)\right)$, define

$$
\psi_{\varepsilon}(y)= \begin{cases}\varphi\left(\frac{y-\varepsilon}{\varepsilon}\right) & \text { for } 0 \leq y \leq \varepsilon \\ \varphi\left(\frac{y-\varepsilon}{L_{2}}\right) & \text { for } y \geq \varepsilon\end{cases}
$$

and let $u_{0}$ be defined by

$$
u_{0}(x, y)=\mu y+C_{1} \varepsilon^{\kappa} \varphi\left(\frac{x}{\varepsilon}\right) \psi_{\varepsilon}(y)
$$

Next fix $0<\rho<L_{1}$ and let $\mu_{0}=\mu_{0}(p, q, \Omega, \rho)>0$ and $c=c(p, q, \Omega, \rho)>0$ be given by Lemmas 3.2 and 3.3. For any $\mu \in\left(0, \mu_{0}\right]$, there exists $\varepsilon_{0}=\varepsilon_{0}(p, q, \Omega, \mu, \rho)>0$ such that, for all $\varepsilon \in\left(0, \varepsilon_{0}\right]$, the function $u_{0} \in \mathcal{V}_{\mu}$ and satisfies

$$
\begin{aligned}
& u_{0} \text { is symmetric with respect to the line } x=0, \\
& \partial_{x} u_{0} \leq 0 \text { in } \Omega_{+}, \\
& \partial_{y} u_{0} \geq \mu / 2 \quad \text { in } \Omega, \\
& u_{0}(x, y) \leq \mu\left(y+c \chi_{(-\rho / 2, \rho / 2) \times\left(0, L_{2}\right)}\right) \quad \text { in } \Omega, \\
& u_{0}(x, y) \geq C_{1} \varepsilon^{\kappa} \quad \text { in } B_{\varepsilon / 3}(0, \varepsilon) \subset \Omega .
\end{aligned}
$$

Proof. Assume $\varepsilon \leq \min \left(L_{1}, L_{2} / 12\right)$. Then

$$
\psi_{\varepsilon}(y)=0 \quad \text { for } y \notin\left[\frac{\varepsilon}{3}, \frac{3 L_{2}}{4}\right]
$$


(indeed, $y \geq \frac{3 L_{2}}{4}$ implies $\frac{y-\varepsilon}{L_{2}} \geq \frac{3}{4}-\frac{1}{12}=\frac{2}{3}$ ) and therefore $u_{0} \in \mathcal{V}_{\mu}$. Properties (6.3)-(6.4) are clear by the choice of $\varphi$.

To check (6.5), we note that

$$
\partial_{y} u_{0}=\mu+C_{1} \varepsilon^{\kappa} \varphi\left(\frac{x}{\varepsilon}\right) \psi_{\varepsilon}^{\prime}(y) .
$$

For $0 \leq y \leq \varepsilon$, we have $\psi_{\varepsilon}^{\prime}(y) \geq 0$, hence $\partial_{y} u_{0} \geq \mu$. Whereas, for $y \geq \varepsilon$, we have

$$
\psi_{\varepsilon}^{\prime}(y)=L_{2}^{-1} \varphi^{\prime}\left((y-\varepsilon) / L_{2}\right) \geq-L_{2}^{-1}\left\|\varphi^{\prime}\right\|_{\infty},
$$

hence

$$
\partial_{y} u_{0} \geq \mu-C_{1} \varepsilon^{\kappa} L_{2}^{-1}\left\|\varphi^{\prime}\right\|_{\infty} \geq \mu / 2
$$

whenever $\varepsilon^{\kappa} \leq \mu L_{2} /\left(2 C_{1}\left\|\varphi^{\prime}\right\|_{\infty}\right)$.

As for (6.6), if $C_{1} \varepsilon^{\kappa} \leq \mu c$ and $\varepsilon \leq \rho / 2$, it immediately follows from $\varphi, \psi_{\varepsilon} \leq 1$ and $\operatorname{supp}(\varphi) \subset(-1,1)$. Finally, since $\varphi(x / \varepsilon)=1$ for $|x| \leq \varepsilon / 3$ and $\psi_{\varepsilon}(y)=1$ for $|y-\varepsilon| \leq \varepsilon / 3$, we have (6.7). The lemma is proved.

Proof of Theorem 1.1 $(i)$. Let $\mu$ and $u_{0}$ be as in Lemma 6.1

- The fact that $T_{\max }\left(u_{0}\right)<\infty$ follows from Lemma 4.1.

- Next, we have $G B U S\left(u_{0}\right) \subset[-\rho, \rho] \times\{0\}$ as a consequence of Lemma 5.3 .

- Properties (1.9)-(1.10) follow from Lemma 3.1.

- Finally, property (1.11) is a consequence of Lemma 3.3(ii).

This proves the assertion.

\section{$7 \quad$ Nondegeneracy of gradient blow-up points}

In this section, we show that if $u$ is only "weakly singular" in a neighborhood of a boundary point $\left(x_{0}, 0\right)$, then the singularity is removable.

Lemma 7.1. Let $\rho, \mu, u_{0}$ be as in Lemma 3.3(ii) and let $x_{0} \in\left(-L_{1}, L_{1}\right)$. There exist $c_{0}=c_{0}(p, q)>0$ such that, if $u_{0} \in \mathcal{V}_{\mu}$ with $T:=T_{\max }\left(u_{0}\right)<\infty$ and

$$
u(x, y) \leq c_{0} y^{(q-p) /(q-p+1)} \quad \text { in } \quad\left(B_{R}\left(x_{0}, 0\right) \cap \Omega\right) \times\left[t_{0}, T\right),
$$

for some $R>0$ and $t_{0} \in(0, T)$, then $\left(x_{0}, 0\right)$ is not a gradient blow-up point.

Proof. Let $x_{0} \in\left(-L_{1}, L_{1}\right)$. Then for some constants $r \in(0, R)$ and $d \in\left(0, L_{2}\right)$, we have that

$$
\omega_{1}:=\left\{(x, y) \in \mathbb{R}^{2} ;\left|x-x_{0}\right|<r, 0<y<d\right\} \subset B_{R}\left(x_{0}, 0\right) \cap \Omega .
$$

Setting $\beta=1 /(q-p+1)$, we define the comparison function

$$
v=v(x, y, t)=\varepsilon y V^{-\beta} \quad \text { in } Q:=\overline{\omega_{1}} \times\left(t_{0}, T\right)
$$

with

$$
V=y+\eta\left(r^{2}-\left(x-x_{0}\right)^{2}\right)\left(t-t_{0}\right),
$$

where $\eta, \varepsilon>0$ are to be determined later. We compute, in $Q$,

$$
v_{t}=-\varepsilon \beta \eta y\left(r^{2}-\left(x-x_{0}\right)^{2}\right) V^{-\beta-1},
$$




$$
\begin{gathered}
v_{x}=2 \varepsilon \beta \eta y\left(x-x_{0}\right)\left(t-t_{0}\right) V^{-\beta-1}, \\
v_{y}=\varepsilon V^{-\beta}-\varepsilon \beta y V^{-\beta-1}=\varepsilon V^{-\beta}\left[1-\beta \frac{y}{V}\right], \\
v_{x x}=2 \varepsilon \beta \eta y\left(t-t_{0}\right) V^{-\beta-1}-4 \varepsilon \beta \eta^{2} y\left(x-x_{0}\right)^{2}\left(t-t_{0}\right)^{2}(-\beta-1) V^{-\beta-2} \\
=2 \varepsilon \beta \eta\left(t-t_{0}\right) V^{-\beta-1}\left[y+2(\beta+1) \eta\left(x-x_{0}\right)^{2}\left(t-t_{0}\right) \frac{y}{V}\right], \\
v_{y y}=-2 \varepsilon \beta V^{-\beta-1}+\varepsilon \beta(\beta+1) y V^{-\beta-2}=\varepsilon \beta V^{-\beta-1}\left[-2+(\beta+1) \frac{y}{V}\right], \\
v_{x y}=2 \varepsilon \beta \eta\left(x-x_{0}\right)\left(t-t_{0}\right) V^{-\beta-1}-2 \varepsilon \beta(\beta+1) \eta y\left(x-x_{0}\right)\left(t-t_{0}\right) V^{-\beta-2} \\
=2 \varepsilon \beta \eta\left(x-x_{0}\right)\left(t-t_{0}\right) V^{-\beta-1}\left[1-(\beta+1) \frac{y}{V}\right] .
\end{gathered}
$$

Noting that $\beta<1$ and $\frac{y}{V} \leq 1$, we see that, in $Q$,

$$
0 \leq v_{x x} \leq 2 \varepsilon \beta \eta T\left(d+2(\beta+1) \eta r^{2} T\right) V^{-\beta-1}, \quad\left|v_{x y}\right| \leq 2 \varepsilon \beta \eta r T V^{-\beta-1}
$$

and

$$
v_{y y} \leq \varepsilon \beta(\beta-1) V^{-\beta-1}<0 .
$$

It follows that

$$
\begin{aligned}
\Delta_{p} v & =|\nabla v|^{p-2}\left[\Delta v+(p-2) \frac{v_{i} v_{j} v_{i j}}{|\nabla v|^{2}}\right] \leq|\nabla v|^{p-2}\left[(p-1) v_{x x}+v_{y y}+(p-2)\left|v_{x y}\right|\right] \\
& \leq \varepsilon \beta|\nabla v|^{p-2} V^{-\beta-1}\left[2(p-1) \eta T\left(d+2(\beta+1) \eta r^{2} T\right)+(\beta-1)+2(p-2) \eta r T\right] .
\end{aligned}
$$

On the other hand, we have

$$
|\nabla v| \geq\left|v_{y}\right| \geq \varepsilon(1-\beta) V^{-\beta} \geq \varepsilon(1-\beta)\left(d+\eta T r^{2}\right)^{-\beta},
$$

hence

$$
v_{t} \geq-\varepsilon \beta \eta d r^{2} V^{-\beta-1} \geq-\varepsilon \beta|\nabla v|^{p-2} V^{-\beta-1}\left[\eta d r^{2}((1-\beta) \varepsilon)^{2-p}\left(d+\eta T r^{2}\right)^{(p-2) \beta}\right] .
$$

Therefore,

$$
\begin{aligned}
& v_{t}-\Delta_{p} v \geq \varepsilon \beta|\nabla v|^{p-2} V^{-\beta-1} \times\left[-\eta d r^{2}((1-\beta) \varepsilon)^{2-p}\left(d+\eta T r^{2}\right)^{(p-2) \beta}\right. \\
&\left.-2(p-1) \eta T\left(d+2(\beta+1) \eta r^{2} T\right)-2(p-2) \eta r T+(1-\beta)\right] .
\end{aligned}
$$

Since also

$$
\left|v_{x}\right| \leq 2 \varepsilon \beta \eta r T V^{-\beta}, \quad\left|v_{y}\right| \leq \varepsilon V^{-\beta},
$$

if we choose $\eta=\eta(p, q, d, r, T, \varepsilon)>0$ small enough, we get that, in $Q$,

$$
|\nabla v| \leq 2 \varepsilon V^{-\beta}
$$


and

$$
v_{t}-\Delta_{p} v \geq \frac{\varepsilon \beta(1-\beta)}{2}|\nabla v|^{p-2} V^{-\beta-1}
$$

hence

$$
v_{t}-\Delta_{p} v \geq \frac{\varepsilon \beta(1-\beta)}{2}|\nabla v|^{p-2}(2 \varepsilon)^{-\frac{\beta+1}{\beta}}|\nabla v|^{\frac{\beta+1}{\beta}}=\frac{\beta(1-\beta)}{4}(2 \varepsilon)^{-\frac{1}{\beta}}|\nabla v|^{q},
$$

due to $\beta=1 /(q-p+2)$. If $\varepsilon=\varepsilon_{0}(p, q)>0$ is small enough, we thus obtain

$$
v_{t}-\Delta_{p} v \geq|\nabla v|^{q}
$$

Now we shall check the comparison on the parabolic boundary of $\omega_{1} \times\left(t_{0}, T\right)$. On $\omega_{1} \times\left\{t_{0}\right\}$, choosing $c_{0}=2^{-\beta} \varepsilon_{0}$, we have

$$
u \leq c_{0} y^{1-\beta}=2^{-\beta} \varepsilon_{0} y^{1-\beta} \leq v .
$$

On the lateral boundary part $\left\{(x, y) \in \mathbb{R}^{2} ;\left|x-x_{0}\right|=r, 0 \leq y \leq d\right\} \times\left(t_{0}, T\right)$, inequality (17.3) holds also. On the surface $\left\{(x, y) \in \mathbb{R}^{2} ;\left|x-x_{0}\right| \leq r, y=0\right\} \subset \partial \Omega$, we have for $t_{0}<t<T$

$$
u(., ., t)=v(., ., t)=0
$$

Finally, on $\left\{(x, y) \in \mathbb{R}^{2} ;\left|x-x_{0}\right| \leq r, y=d\right\} \times\left(t_{0}, T\right)$, assuming in addition that $\eta$ satisfies $\eta \leq d T^{-1} r^{-2}$, we get

$$
u \leq c_{0} d^{1-\beta} \leq \varepsilon_{0} d\left(d+\eta r^{2} T\right)^{-\beta} \leq v .
$$

Using the comparison principle, we get that

$$
u \leq v \quad \text { in } \omega_{1} \times\left(t_{0}, T\right) .
$$

This implies that

$$
\left|u_{y}\right| \leq \varepsilon\left(\eta\left(r^{2}-\left|x-x_{0}\right|^{2}\right)\left(t-t_{0}\right)\right)^{-\beta} \leq M_{0}
$$

on $\left(B_{r / 2}\left(\left(x_{0}, 0\right)\right) \cap \partial \Omega\right) \times\left(\left(t_{0}+T\right) / 2, T\right)$ for some constant $M_{0}>0$. Lemma 7.1 is then a direct consequence of Lemma 5.1 .

\section{The auxiliary function $J$ and the proof of single- point gradient blow-up}

In all this section, we fix $\rho, x_{1}$ with

$$
0<\rho<x_{1}<L_{1}
$$

and we assume that $\mu$ and $u_{0} \in \mathcal{V}_{\mu} \cap C^{2}(\bar{\Omega})$ satisfy the assumption of Theorem 1.1(ii) i.e., the corresponding solution of (1.1) fulfills properties (1.8)-(1.11). We denote as before $T=T_{\max }\left(u_{0}\right)$.

We consider the auxiliary function

$$
J(x, y, t):=u_{x}+c(x) d(y) F(u),
$$


with

$$
\left\{\begin{array}{l}
F(u)=u^{\alpha} \\
c(x)=k x, \quad k>0 \\
d(y)=y^{-\gamma}
\end{array}\right.
$$

and

$$
1<\alpha<1+q-p, \quad \gamma=(1-2 \sigma)(\alpha-1),
$$

where

$$
0<\sigma<\frac{1}{2(q-p+1)}
$$

is fixed. Letting

$$
D=\left(0, x_{1}\right) \times\left(0, y_{1}\right),
$$

our goal is to use a comparison principle to prove that

$$
J \leq 0 \quad \text { in } D \times(T / 2, T),
$$

provided $\alpha>1$ is chosen close enough to 1 (hence making $\gamma>0$ small) and $y_{1} \in\left(0, L_{2}\right)$ and $k>0$ are chosen sufficiently small.

\subsection{Parabolic inequality for the auxiliary function $J$}

By the regularity of $u$ (see Theorem 2.2), we have

$$
J \in C^{2,1}\left(Q_{T}\right) .
$$

A key step is to derive a parabolic inequality for $J$. To this end, we define the operator

$$
\mathcal{P} J:=J_{t}-|\nabla u|^{p-2} \Delta J-(p-2)|\nabla u|^{p-4}\left\langle D^{2} J \nabla u, \nabla u\right\rangle+\mathcal{H} \cdot \nabla J+\mathcal{A} J,
$$

where the functions $\mathcal{H}=\mathcal{H}(x, y, t)$ and $\mathcal{A}=\mathcal{A}(x, y, t)$ are given by formulae (9.9)-(9.12) below.

Proposition 8.1. Assume (8.1), (8.3) and let $\mu, u_{0}$ satisfy the assumption of Theorem 1.1(ii). There exist $\alpha, \gamma$ satisfying (8.2), $y_{1} \in\left(0, L_{2}\right)$ and $k_{0}>0$, all depending only on $p, q, \Omega, \mu,\left\|u_{0}\right\|_{C^{2}}, \sigma$, such that, for any $k \in\left(0, k_{0}\right]$, the function $J$ satisfies

$$
\mathcal{P} J \leq 0 \quad \text { in } D \times(T / 2, T)
$$

Moreover,

$$
\mathcal{H}, \mathcal{A} \in C(D \times(0, T)) \quad \text { and } \quad \mathcal{A} \in L^{\infty}(D \times(T / 2, \tau)) \quad \text { for each } \tau \in(T / 2, T) .
$$

The proof of Proposition 8.1 is very long and technical. In order not to disrupt the main line of argument, we postpone it to section 9 and now present the rest of the proof of Theorem 1.1(ii). 


\subsection{Boundary conditions for the auxiliary function $J$}

The verification of the appropriate boundary and initial conditions for the function $J$ depends on an essential way on the applicability to $u_{x}$ of the Hopf boundary lemma at the points $\left(x_{1}, 0\right)$ and $\left(0, y_{1}\right)$, up to $t=T$. To this end, besides the nondegeneracy of problem (1.1), guaranteed by (1.11), we also need the following local regularity lemma, which ensures that $D^{2} u$ remains bounded up to $t=T$ away from the gradient blow-up set.

Lemma 8.2. Let $\rho \in\left(0, L_{1}\right)$ and let $\mu, u_{0}$ satisfy the assumption of Theorem 1.1(ii). Let $\omega^{\prime} \subset \omega \subset \Omega$ be such that $\operatorname{dist}\left(\omega^{\prime}, \Omega \backslash \omega\right)>0$ and $t_{0} \in(0, T)$. If

$$
\sup _{\omega \times(0, T)}|\nabla u|<\infty
$$

then

$$
\sup _{\omega^{\prime} \times\left(t_{0}, T\right)}\left|D^{2} u\right|<\infty .
$$

Proof. Introduce an intermediate domain $\omega^{\prime \prime}$ with $\omega^{\prime} \subset \omega^{\prime \prime} \subset \omega$, such that $\operatorname{dist}\left(\omega^{\prime \prime}, \Omega \backslash \omega\right)>$ 0 and $\operatorname{dist}\left(\omega^{\prime}, \Omega \backslash \omega^{\prime \prime}\right)>0$. Write the PDE in (1.1) as

$$
-\nabla \cdot\left(|\nabla|^{p-2} \nabla u\right)=|\nabla u|^{q}-u_{t} .
$$

Using $|\nabla u| \geq \partial_{y} u \geq \mu / 2$ (cf. (1.11)) and the boundedness of $u_{t}$ in $Q_{T}$ (cf. Lemma 3.4), it follows from the elliptic estimate in [13, Theorem V.5.2] that there exists $\theta \in(0,1)$ such that

$$
\|\nabla u(\cdot, t)\|_{C^{\theta}\left(\bar{\omega}^{\prime \prime}\right)} \leq C, \quad t_{0} / 2 \leq t<T .
$$

The boundedness of $u_{t}$ in $Q_{T}$ and the interpolation result in [13, Lemma II.3.1] then guarantee the estimate

$$
\|\nabla u(x, y, \cdot)\|_{C^{\beta}\left(\left[t_{0} / 2, T\right)\right)} \leq C, \quad(x, y) \in \bar{\omega}^{\prime},
$$

where $\beta=\theta /(1+\theta)$. Therefore $\|\nabla u\|_{C^{\beta}\left(\bar{\omega}^{\prime} \times\left[t_{0} / 2, T\right)\right)} \leq C$. The conclusion now follows by applying standard Schauder parabolic estimates to the PDE in (1.1), rewritten under the form

$$
u_{t}-a_{i j} u_{i j}=f, \quad \text { where } a_{i j}=|\nabla u|^{p-2}\left[\delta_{i j}+(p-2) \frac{u_{i} u_{j}}{|\nabla u|^{2}}\right], \quad f=|\nabla u|^{q} .
$$

Indeed, the matrix $\left(a_{i j}\right)=\left(a_{i j}(x, y, t)\right)$ is uniformly elliptic due to (1.11) and

$$
\begin{aligned}
a_{i j} \xi_{i} \xi_{j} & =|\nabla u|^{p-2}|\xi|^{2}+(p-2)|\nabla u|^{p-4}|\nabla u \cdot \xi|^{2} \\
& \geq|\nabla u|^{p-2}|\xi|^{2} \geq(\mu / 2)^{p-2}|\xi|^{2}
\end{aligned}
$$

and there exists $\nu \in(0,1)$ such that $a_{i j}, f \in C^{\nu}\left(\bar{\omega}^{\prime} \times\left[t_{0} / 2, T^{\prime}\right]\right)$, for each $T^{\prime}<T$, with norm independent of $T^{\prime}$.

Lemma 8.3. Assume (8.1)-(8.3), let $y_{1} \in\left(0, L_{2}\right)$ and let $\mu, u_{0}$ satisfy the assumption of Theorem 1.1(ii). Then

$$
J \in C(\bar{D} \times(0, T))
$$

and there exists $k_{1}>0$ (depending in particular on $y_{1}$ ) such that, for any $k \in\left(0, k_{1}\right]$, the function $J$ satisfies

$$
J \leq 0 \quad \text { on } \partial D \times(T / 2, T)
$$


Proof. Since $u=0$ for $y=0$ and $|\nabla u| \leq C(\tau)$ in $\Omega \times[0, \tau]$ for each $\tau<T$, we have

$$
u \leq C(\tau) y \quad \text { in } D \times[0, \tau] .
$$

Due to $\gamma<\alpha$, we may therefore extend the function $c(x) d(y) F(u)$ continuously to be 0 for $y=0$. Property (8.8) then follows from the regularity of $u$ (see Theorem 2.2) and we have

$$
J=0 \quad \text { on }\left(0, x_{1}\right) \times\{0\} \times(T / 2, T) .
$$

By (1.10), we have

$$
u_{x}=0 \quad \text { on }\{0\} \times\left(0, y_{1}\right) \times(0, T),
$$

hence

$$
J=0 \text { on }\{0\} \times\left(0, y_{1}\right) \times(T / 2, T) .
$$

Next, the function $w=u_{x}$ is $\leq 0$ in $\Omega^{+} \times(0, T)$ (cf. (1.10) $)$ and satisfies there:

$$
w_{t}=a_{i j}(x, y, t) w_{i j}+B(x, y, t) \cdot \nabla w,
$$

with

$$
\begin{aligned}
a_{i j}(x, y, t) & =|\nabla u|^{p-2}\left[\delta_{i j}+(p-2) \frac{u_{i} u_{j}}{|\nabla u|^{2}}\right], \\
B(x, y, t) & =(p-2)|\nabla u|^{p-4} \nabla u \Delta u+(p-2)(p-4)|\nabla u|^{p-6}\left\langle D^{2} u \nabla u, \nabla u\right\rangle \nabla u \\
& +q|\nabla u|^{q-2} \nabla u+2(p-2)|\nabla u|^{p-4}\left(D^{2} u \nabla u\right) .
\end{aligned}
$$

Fix $\rho<x_{3}<x_{2}<x_{1}$. Since $G B U S \subset[-\rho, \rho] \times\{0\}$, we have

$$
|\nabla u| \leq C \quad \text { in }\left(\Omega \backslash\left\{\left(-x_{3}, x_{3}\right) \times\left(0, y_{1} / 3\right)\right\}\right) \times(0, T) .
$$

It follows from Lemma 8.2 that

$$
\left|D^{2} u\right| \leq C \quad \text { in }\left(\Omega \backslash\left\{\left(-x_{2}, x_{2}\right) \times\left(0, y_{1} / 2\right)\right\}\right) \times(T / 4, T),
$$

hence

$$
|B| \leq C \quad \text { in }\left(\Omega \backslash\left\{\left(-x_{2}, x_{2}\right) \times\left(0, y_{1} / 2\right)\right\}\right) \times(T / 4, T) .
$$

Moreover, the matrix $A(x, y, t)$ is uniformly elliptic (cf. (8.7)). We may thus apply the strong maximum principle and the Hopf boundary point Lemma [19, Theorem 6 p. 174], to get

$$
\begin{aligned}
& u_{x} \leq-c_{1} y \text { on }\left\{x_{1}\right\} \times\left(0, y_{1}\right) \times(T / 2, T), \\
& u_{x} \leq-c_{1} x \text { on }\left(0, x_{1}\right) \times\left\{y_{1}\right\} \times(T / 2, T) .
\end{aligned}
$$

Also, since $x_{1}>\rho$ and $u(x, 0, t)=0$, we get that, for some $c_{2}>0$,

$$
u \leq c_{2} y \quad \text { on }\left\{x_{1}\right\} \times\left(0, y_{1}\right) \times(T / 2, T) .
$$

Consequently, using $\alpha>\gamma+1$ and (2.6) , we have for $0<k \leq k_{1}\left(y_{1}\right)$ sufficiently small

$$
\begin{aligned}
& J\left(x, y_{1}, t\right) \leq-c_{1} x+k x y_{1}^{-\gamma}\left\|u_{0}\right\|_{\infty}^{\alpha} \leq 0 \quad \text { on }\left(0, x_{1}\right) \times\left\{y_{1}\right\} \times(T / 2, T), \\
& J\left(x_{1}, y, t\right) \leq-c_{1} y+k x_{1} y^{\alpha-\gamma} c_{2}^{\alpha} \leq 0 \quad \text { on }\left\{x_{1}\right\} \times\left(0, y_{1}\right) \times(T / 2, T) .
\end{aligned}
$$

This, along with (8.10)-(8.11), proves (8.9). 


\subsection{Initial conditions for $J$}

Lemma 8.4. Assume (8.1)-(8.3) and let $\mu, u_{0}$ satisfy the assumption of Theorem 1.1(ii). There exists $k_{2}>0$ such that, for any $k \in\left(0, k_{2}\right]$, the function $J$ satisfies

$$
J(x, y, T / 2) \leq 0 \quad \text { in }\left[0, x_{1}\right] \times\left[0, L_{2}\right] .
$$

The proof relies on a parabolic version of the Serrin corner lemma applied to $u_{x}$. This is provided by Proposition 11.1, which we state and prove in Appendix 2.

Proof. The function $z=u_{x}$ satisfies equation (8.12). We shall apply Proposition 11.1 to this equation, with $\tau_{1}=T / 4, \tau_{2}=3 T / 4, X_{1}=x_{1}, Y_{1}=L_{2}, \hat{X}_{1}=L_{1}, \hat{Y}_{1}=2 L_{2}$. We thus need to check the assumption (11.2) . Let us denote $\hat{D}_{T}=\left(0, \hat{X}_{1}\right) \times\left(0, \hat{Y}_{1}\right) \times(T / 4,3 T / 4)$.

For $x=0$ or $y=0$, we have $u_{x}=0$, hence $a_{12}=a_{21}=(p-2)|\nabla u|^{p-2} u_{x} u_{y}=0$. Due to the regularity of $u$ (cf. (2.7) $)$, we deduce that

$$
a_{12}+a_{21} \geq-C(x \wedge y) \quad \text { in } \overline{\hat{D}_{T}}
$$

On the other hand, for $x=0$ and $0<y<\hat{Y}_{1}$, we have $u_{x y}=u_{x}=0$. Also, by (2.9), we have $\left|\left(u_{x y}\right)_{x}\right|=\left|\left(u_{x}\right)_{y x}\right| \leq C$ in $\overline{\hat{D}_{T}}$. Consequently $\left|u_{x}\right|+\left|u_{x y}\right| \leq C x$ in $\overline{\hat{D}_{T}}$. Using (2.7) and (1.11), we deduce

$$
\begin{aligned}
B_{1}= & (p-2)|\nabla u|^{p-4}(\Delta u) u_{x}+(p-2)(p-4)|\nabla u|^{p-6}\left\langle D^{2} u \nabla u, \nabla u\right\rangle u_{x} \\
& +q|\nabla u|^{q-2} u_{x}+2(p-2)|\nabla u|^{p-4}\left(u_{x x} u_{x}+u_{x y} u_{y}\right) \\
\geq & -C x \quad \text { in } \overline{\hat{D}_{T}}
\end{aligned}
$$

Next, for $y=0$ and $0<x<\hat{X}_{1}$, we have $u_{t}=0$ and $u_{x}=u_{x x}=0$. Recalling (2.7), we thus have

$$
\begin{aligned}
\left(u_{y}\right)^{q} & =|\nabla u|^{q}=u_{t}-\Delta_{p} u=-|\nabla u|^{p-2}\left[\Delta u+(p-2) \frac{\left\langle D^{2} u \nabla u, \nabla u\right\rangle}{|\nabla u|^{2}}\right] \\
& =-|\nabla u|^{p-2}\left[u_{x x}+u_{y y}+(p-2) \frac{u_{x x} u_{x}^{2}+2 u_{x y} u_{x} u_{y}+u_{y y} u_{y}^{2}}{u_{x}^{2}+u_{y}^{2}}\right]=-(p-1)\left(u_{y}\right)^{p-2} u_{y y} .
\end{aligned}
$$

It follows that, for $0<x<\hat{X}_{1}$ and $t \in[T / 4,3 T / 4]$,

$$
\begin{aligned}
B_{2}(x, 0, t)= & (p-2)(p-4)|\nabla u|^{p-6}\left(u_{x x} u_{x}^{2}+2 u_{x y} u_{x} u_{y}+u_{y y} u_{y}^{2}\right) u_{y} \\
& +(p-2)|\nabla u|^{p-4}\left(u_{x x}+u_{y y}\right) u_{y}+q|\nabla u|^{q-2} u_{y} \\
& +2(p-2)|\nabla u|^{p-4}\left(u_{y x} u_{x}+u_{y y} u_{y}\right) \\
= & (p-2)(p-4)\left(u_{y}\right)^{p-3} u_{y y}+(p-2)\left(u_{y}\right)^{p-3} u_{y y} \\
& +q\left(u_{y}\right)^{q-1}+2(p-2)\left(u_{y}\right)^{p-3} u_{y y} \\
= & (p-2)(p-1)\left(u_{y}\right)^{p-3} u_{y y}+q\left(u_{y}\right)^{q-1}=(q+2-p)\left(u_{y}\right)^{q-1} \\
\geq & (q+2-p)(\mu / 2)^{q-1}>0 .
\end{aligned}
$$

Therefore, owing to (2.7), there exists $\eta>0$ such that

$$
B_{2}(x, y, t) \geq 0 \quad \text { on }\left(0, \hat{X}_{1}\right) \times[0, \eta] \times[T / 4,3 T / 4],
$$


which implies

$$
B_{2} \geq-C y \quad \text { in } \overline{\hat{D}_{T}}
$$

In view of (8.13)-(8.15), we may thus apply Proposition 11.1 to deduce

$$
u_{x}(x, y, T / 2) \leq-c_{3} x y \quad \text { in }\left[0, x_{1}\right] \times\left[0, L_{2}\right]
$$

Let $C:=\|\nabla u(\cdot, T / 2)\|_{\infty}$. Since $\alpha>\gamma+1$, we get that, for $k \in\left(0, k_{2}\right]$ with $k_{2}>0$ small enough,

$$
J(x, y, T / 2) \leq-c_{3} x y+k x C^{\alpha} y^{\alpha-\gamma} \leq\left[k C^{\alpha} L_{2}^{\alpha-\gamma-1}-c_{3}\right] x y \leq 0 \quad \text { in }\left[0, x_{1}\right] \times\left[0, L_{2}\right] .
$$

\subsection{Proof of Theorem 1.1(ii)}

Let $\alpha, \gamma, y_{1}, k_{0}$ be given by Proposition 8.1 and let $k_{1}, k_{2}$ be given by Lemmas 8.3 8.4. We take $k=\min \left(k_{0}, k_{1}, k_{2}\right)$. By these results and the maximum principle, we have

$$
J \leq 0 \quad \text { in } D \times(T / 2, T) .
$$

Integrating inequality (8.16) over $(0, x)$ for $0<x<x_{1}$, with fixed $y$, we get that

$$
u \leq C x^{-2 /(\alpha-1)} y^{1-2 \sigma} \quad \text { in } D \times(T / 2, T),
$$

where $C=C(\alpha, k, \sigma)>0$. Using that $1-2 \sigma>\frac{q-p}{q-p+1}$, it follows from the nondegeneracy property in Lemma 7.1 that no point $\left(x_{0}, 0\right)$ with $0<\left|x_{0}\right| \leq \rho$ can be a gradient blow-up point. In view of (1.8), we conclude that $G B U S\left(u_{0}\right)=\{(0,0)\}$.

\section{Proof of the main parabolic inequality (Proposi- tion 8.1)}

The proof is quite technical. For sake of clarity, some of the intermediate calculations will be summarized in Lemma 9.1 and 9.2 below.

We first compute

$$
\begin{aligned}
J_{t} & =u_{x t}+c d F^{\prime}(u) u_{t} \\
& =\left(\Delta_{p} u\right)_{x}+\left(|\nabla u|^{q}\right)_{x} \underbrace{+c d F^{\prime} \Delta_{p} u}_{\left(0_{p}\right)}+\underbrace{c d F^{\prime}|\nabla u|^{q}}_{\left(0_{q}\right)} .
\end{aligned}
$$

and

$$
\begin{aligned}
\left(\Delta_{p} u\right)_{x} & =|\nabla u|^{p-2} \Delta\left(u_{x}\right) \\
& +(p-2) \Delta u|\nabla u|^{p-4} \nabla u \cdot \nabla u_{x} \\
& +(p-2)|\nabla u|^{p-4}\left\langle D^{2} u_{x} \nabla u, \nabla u\right\rangle \\
& +(p-2)(p-4)|\nabla u|^{p-6} \nabla u \cdot \nabla u_{x}\left\langle D^{2} u \nabla u, \nabla u\right\rangle \\
& +2(p-2)|\nabla u|^{p-4}\left\langle D^{2} u \nabla u, \nabla u_{x}\right\rangle .
\end{aligned}
$$


Using that $u_{x}=J-c d F(u)$, we write

$$
\begin{aligned}
& \nabla u_{x}=\nabla J-c d F^{\prime} \nabla u-F\left(\begin{array}{c}
c^{\prime} d \\
d^{\prime} c
\end{array}\right) \\
& D^{2} u_{x}=D^{2} J-c d F^{\prime} D^{2} u-c d F^{\prime \prime}\left(\begin{array}{cc}
u_{x}^{2} & u_{x} u_{y} \\
u_{x} u_{y} & u_{y}^{2}
\end{array}\right) \\
& -F(u)\left(\begin{array}{cc}
c^{\prime \prime} d & c^{\prime} d^{\prime} \\
c^{\prime} d^{\prime} & d^{\prime \prime} c
\end{array}\right) \\
& -F^{\prime}(u)\left(\begin{array}{cc}
2 c^{\prime} d u_{x} & c d^{\prime} u_{x}+c^{\prime} d u_{y} \\
c d^{\prime} u_{x}+c^{\prime} d u_{y} & 2 c d^{\prime} u_{y}
\end{array}\right) \\
& \Delta u_{x}=\operatorname{Trace}\left(D^{2} u_{x}\right)=\Delta J-c d F^{\prime} \Delta u-c d F^{\prime \prime}|\nabla u|^{2}-F\left[c^{\prime \prime} d+d^{\prime \prime} c\right] \\
& -2 F^{\prime} c^{\prime} d J+2 F^{\prime} F c^{\prime} c d^{2}-2 F^{\prime}(u) d^{\prime} c u_{y}, \\
& \left\langle D^{2} u_{x}, \nabla u, \nabla u\right\rangle=\left\langle D^{2} J, \nabla u, \nabla u\right\rangle-c d F^{\prime \prime}|\nabla u|^{4}-c d F^{\prime}\left\langle D^{2} u, \nabla u, \nabla u\right\rangle-F c^{\prime \prime} d u_{x}^{2} \\
& -2 F c^{\prime} d^{\prime} u_{x} u_{y}-F c d^{\prime \prime} u_{y}^{2} \\
& -2 F^{\prime}|\nabla u|^{2}\left(c d^{\prime} u_{y}+d c^{\prime} u_{x}\right)
\end{aligned}
$$

and

$$
\begin{aligned}
\left\langle D^{2} u, \nabla u, \nabla u_{x}\right\rangle= & \left\langle D^{2} u, \nabla u, \nabla J\right\rangle-c d F^{\prime}\left\langle D^{2} u, \nabla u, \nabla u\right\rangle \\
& -F c^{\prime} d \nabla u \cdot \nabla J+c c^{\prime} d^{2} F^{\prime} F|\nabla u|^{2}+d^{2} F^{2}\left(c^{\prime}\right)^{2} J \\
& -d^{2} F^{2}\left(c^{\prime}\right)^{2} c d F+c^{\prime} c d^{\prime} d F^{2} u_{y}-F d^{\prime} c\left(u_{x} u_{x y}+u_{y} u_{y y}\right) .
\end{aligned}
$$

Therefore,

$$
\begin{aligned}
\left(\Delta_{p} u\right)_{x}= & |\nabla u|^{p-2}\left[\Delta J-c d F^{\prime} \Delta u-c d F^{\prime \prime}|\nabla u|^{2}-2 c^{\prime} d F^{\prime} J\right. \\
& \left.+2 c c^{\prime} d^{2} F F^{\prime}-2 d^{\prime} c F^{\prime} u_{y}-F\left(c^{\prime \prime} d+d^{\prime \prime} c\right)\right] \\
+ & (p-2) \Delta u|\nabla u|^{p-4}\left[\nabla u \cdot \nabla J-c d F^{\prime}|\nabla u|^{2}-u_{y} c d^{\prime} F-c^{\prime} d F J+c^{\prime} c d^{2} F^{2}\right] \\
+ & (p-2)|\nabla u|^{p-4}\left[\left\langle D^{2} J \nabla u, \nabla u\right\rangle-c d F^{\prime}\left\langle D^{2} u \nabla u, \nabla u\right\rangle-c d F^{\prime \prime}|\nabla u|^{4}\right. \\
& -2 c^{\prime} d F^{\prime}|\nabla u|^{2} J+2 c^{\prime} c d^{2} F^{\prime} F|\nabla u|^{2}-2 c d^{\prime} F^{\prime} u_{y}|\nabla u|^{2} \\
& \left.-c^{\prime \prime} d F\left(u_{x}\right)^{2}-2 c^{\prime} d^{\prime} F J u_{y}+2 c^{\prime} c d^{\prime} d F^{2} u_{y}-d^{\prime \prime} c F\left(u_{y}\right)^{2}\right] \\
& \\
+ & (p-2)(p-4)|\nabla u|^{p-6}\left\langle D^{2} u \nabla u, \nabla u\right\rangle[\nabla u \cdot \nabla J \\
& \left.-c d F^{\prime}|\nabla u|^{2}-u_{y} c d^{\prime} F-c^{\prime} d F J+c^{\prime} c d^{2} F^{2}\right] \\
& \\
+ & 2(p-2)|\nabla u|^{p-4}\left[\left\langle D^{2} u \nabla u, \nabla J\right\rangle-c d F^{\prime}\left\langle D^{2} u \nabla u, \nabla u\right\rangle-c^{\prime} d F \nabla u \cdot \nabla J\right. \\
& +c^{\prime} c d^{2} F^{\prime} F|\nabla u|^{2}+\left(c^{\prime} d\right)^{2} F^{2} J-\left(c^{\prime} d\right)^{2} F^{3} c d+c^{\prime} d^{\prime} c d F^{2} u_{y}-d^{\prime} c F u_{y y} u_{y} \\
& \left.-d^{\prime} c F \nabla J \cdot L+d^{\prime} d c^{2} F^{\prime} F u_{y} J-d^{\prime} d^{2} c^{3} F^{\prime} F^{2} u_{y}+\left(d^{\prime} c F\right)^{2} J-\left(d^{\prime} c F\right)^{2} c d F\right],
\end{aligned}
$$


where $L=\left(\begin{array}{c}0 \\ u_{x}\end{array}\right)$. This can be rewritten as

$$
\begin{aligned}
\left(\Delta_{p} u\right)_{x}= & |\nabla u|^{p-2} \Delta J+(p-2)|\nabla u|^{p-4}\left\langle D^{2} J \nabla u, \nabla u\right\rangle+\mathcal{H}_{1} \cdot \nabla J+\mathcal{A}_{1}(x, y, t) J \\
& -F|\nabla u|^{p-2}\left[c^{\prime \prime} d+d^{\prime \prime} c\right]-(p-2) F|\nabla u|^{p-4}\left[c^{\prime \prime} d u_{x}^{2}+d^{\prime \prime} c u_{y}^{2}\right] \\
& \left.-2(p-2) c d F|\nabla u|^{p-4}\left[\left(c^{\prime} d F\right)^{2}+\left(d^{\prime} c F\right)^{2}\right]-(p-1) c d F^{\prime \prime}|\nabla u|^{p}\right\} \quad(1) \\
& +4(p-2) F^{2} c^{\prime} c d^{\prime} d|\nabla u|^{p-4} u_{y} \\
& \left.+(4 p-6) c^{\prime} c d^{2} F^{\prime} F|\nabla u|^{p-2}-2(p-1) c d^{\prime} F^{\prime}|\nabla u|^{p-2} u_{y}\right\} \quad(2) \geq 0 \\
& \left.-2(p-2) d^{\prime} d^{2} F^{\prime} F^{2} c^{3}|\nabla u|^{p-4} u_{y}\right\} \quad \underbrace{-(p-1) c d F^{\prime} \Delta_{p} u}_{(3)} \quad \underbrace{-2(p-2) d^{\prime} c F|\nabla u|^{p-4} u_{y} u_{y y}}_{(5)} \\
& \underbrace{-(p-2) c d^{\prime} F u_{y}\left[|\nabla u|^{p-4} \Delta u+(p-4)|\nabla u|^{p-6}\left\langle D^{2} u \nabla u, \nabla u\right\rangle\right]}_{(6)}
\end{aligned}
$$

where

$$
\begin{aligned}
\mathcal{H}_{1}:= & (p-2)\left[|\nabla u|^{p-4} \Delta u+(p-4)|\nabla u|^{p-6}\left\langle D^{2} u \nabla u, \nabla u\right\rangle\right] \nabla u \\
& -2(p-2) c d^{\prime} F|\nabla u|^{p-4} L \\
& -2(p-2) c^{\prime} d F|\nabla u|^{p-4} \nabla u+2(p-2)|\nabla u|^{p-4}\left(D^{2} u, \nabla u\right)
\end{aligned}
$$

and

$$
\begin{aligned}
\mathcal{A}_{1}:= & -2(p-1) F^{\prime} c^{\prime} d|\nabla u|^{p-2} \\
& -(p-2) F c^{\prime} d\left[|\nabla u|^{p-4} \Delta u+(p-4)|\nabla u|^{p-6}\left\langle D^{2} u \nabla u, \nabla u\right\rangle\right] \\
& +2(p-2)|\nabla u|^{p-4} F^{2}\left[\left(c^{\prime} d\right)^{2}+\left(d^{\prime} c\right)^{2}\right]+2(p-2) d^{\prime} d F^{\prime} F c^{2}|\nabla u|^{p-4} u_{y} \\
& -2(p-2) c^{\prime} d^{\prime} F|\nabla u|^{p-4} u_{y} .
\end{aligned}
$$

On the other hand, we have

$$
\begin{aligned}
\left(|\nabla u|^{q}\right)_{x} & =q|\nabla u|^{q-2} \nabla u \cdot \nabla u_{x} \\
& =q|\nabla u|^{q-2} \nabla u \cdot \nabla J-q c^{\prime} d F|\nabla u|^{q-2} J+(7),
\end{aligned}
$$

where

$$
(7):=\underbrace{-q c d F^{\prime}|\nabla u|^{q}}_{\left(7_{-}\right) \leq 0}+\underbrace{q c c^{\prime} d^{2} F^{2}|\nabla u|^{q-2}-q c d^{\prime} F|\nabla u|^{q-2} u_{y}}_{\left(7_{+}\right) \geq 0} \text {. }
$$

Setting

$$
\mathcal{A}_{2}:=\mathcal{A}_{1}-q c^{\prime} d F|\nabla u|^{q-2}, \quad \mathcal{H}_{2}:=\mathcal{H}_{1}+q|\nabla u|^{q-2} \nabla u,
$$

we have thus proved the following lemma. 
Lemma 9.1. Define the parabolic operator:

$$
\mathcal{L} J:=J_{t}-|\nabla u|^{p-2} \Delta J-(p-2)|\nabla u|^{p-4}\left\langle D^{2} J \nabla u, \nabla u\right\rangle-\mathcal{H}_{2} \cdot \nabla J-\mathcal{A}_{2} J
$$

Then

$$
\mathcal{L} J=\left(0_{p}\right)+\left(0_{q}\right)+(1)+(2)+(3)+(4)+(5)+(6)+(7) .
$$

As a significant difficulty as compared with the semilinear case $p=2$, many additional terms appear in the contributions (1), (2), (4)-(6), and especially nonlinear, second order terms in (4)-(6). To proceed further, we need to observe that, among the second derivatives of $u, u_{y y}$ needs a special treatment, since it is not immediately expressed in terms of $\nabla J$ unlike $u_{x x}$ and $u_{x y}$. Namely we shall eliminate $u_{y y}$ by expressing it in terms of $u_{t}, \nabla u$, $u_{x x}$ and $u_{x y}$ by using the equation. Although this will make the computation even more involved, by producing a lot of additional terms, this seems to be the only way to control the effects of $u_{y y}$. The bound on $u_{t}$ given by Lemma 3.4 will be helpful in this process.

First we have

$$
(3)=-(p-1) c d F^{\prime} \Delta_{p} u=\underbrace{-c d F^{\prime} \Delta_{p} u}_{-\left(0_{p}\right)} \underbrace{-(p-2) c d F^{\prime} u_{t}}_{\left(3_{t}\right)} \underbrace{+(p-2) c d F^{\prime}|\nabla u|^{q}}_{\left(3_{q}\right)} .
$$

To deal with (4), we set

$$
u_{y y}=\frac{u_{t}-|\nabla u|^{q}-\nabla u_{x} \cdot M}{w}
$$

where

$$
M:=\left(\begin{array}{c}
|\nabla u|^{p-2}+(p-2)|\nabla u|^{p-4} u_{x}^{2} \\
2(p-2)|\nabla u|^{p-4} u_{x} u_{y}
\end{array}\right) \quad \text { and } \quad w=|\nabla u|^{p-2}+(p-2)|\nabla u|^{p-4} u_{y}^{2} .
$$

Since $u_{x}=J-c d F$, we get

$$
\begin{aligned}
\nabla u_{x} \cdot M= & -c d F^{\prime} J\left[|(p-1) \nabla u|^{p-2}+(p-2)|\nabla u|^{p-4} u_{y}^{2}\right]-2(p-2) c d^{\prime} F u_{y}|\nabla u|^{p-4} J \\
& +2(p-2) c^{2} d^{\prime} d F^{2} u_{y}|\nabla u|^{p-4}+c^{2} d^{2} F^{\prime} F\left[|(p-1) \nabla u|^{p-2}+(p-2)|\nabla u|^{p-4} u_{y}^{2}\right] \\
& +\nabla J \cdot M-c^{\prime} d F\left[|\nabla u|^{p-2}+(p-2)|\nabla u|^{p-4} u_{x}^{2}\right] .
\end{aligned}
$$

It follows that

$$
\begin{aligned}
u_{y y}= & \frac{u_{t}-|\nabla u|^{q}}{w}-\frac{\nabla J \cdot M}{w}+\frac{c d F^{\prime} J\left[(p-1)|\nabla u|^{p-2}+(p-2)|\nabla u|^{p-4} u_{y}^{2}\right]}{w} \\
& +\frac{2(p-2) c d^{\prime} F u_{y}|\nabla u|^{p-4} J}{w}+\frac{c^{\prime} d F\left[|\nabla u|^{p-2}+(p-2)|\nabla u|^{p-4} u_{x}^{2}\right]}{w} \\
& -\frac{c^{2} d^{2} F F^{\prime}\left[(p-1)|\nabla u|^{p-2}+(p-2)|\nabla u|^{p-4} u_{y}^{2}\right]}{w} \\
& -\frac{2(p-2) c^{2} d^{\prime} d F^{2} u_{y}|\nabla u|^{p-4}}{w} .
\end{aligned}
$$


Now, to treat the contribution of $u_{y y}$ in (5) and (6), we set $N=\left(\begin{array}{c}u_{x}^{2} \\ 2 u_{x} u_{y}\end{array}\right)$ and rewrite

$$
\begin{aligned}
|\nabla u|^{p-4} \Delta u+(p-4)|\nabla u|^{p-6}\left\langle D^{2} u \nabla u, \nabla u\right\rangle & =\frac{\Delta_{p} u}{|\nabla u|^{2}}-2|\nabla u|^{p-6}\left\langle D^{2} u \nabla u, \nabla u\right\rangle \\
& =\frac{u_{t}-|\nabla u|^{q}}{|\nabla u|^{2}}-2|\nabla u|^{p-6}\left[\nabla u_{x} \cdot N+u_{y}^{2} u_{y y}\right] .
\end{aligned}
$$

We have

$$
\begin{aligned}
\nabla u_{x} \cdot N= & \nabla J \cdot N-c d F^{\prime} J\left[u_{x}^{2}+2 u_{y}^{2}\right]+c^{2} d^{2} F F^{\prime}\left[u_{x}^{2}+2 u_{y}^{2}\right]-c^{\prime} d F u_{x}^{2} \\
& -2 c d^{\prime} F J u_{y}+2 d^{\prime} d c^{2} F^{2} u_{y}
\end{aligned}
$$

The expression in (4) then becomes

$$
\begin{aligned}
(4)= & \left.\frac{2(p-2) d^{\prime} c F|\nabla u|^{p-4} u_{y} M \cdot \nabla J}{w}\right\}\left(4_{\nabla}\right) \\
& \left.-\frac{2(p-2) c^{2} d^{\prime} d F^{\prime} F|\nabla u|^{p-4} u_{y}\left[(p-1)|\nabla u|^{p-2}+(p-2)|\nabla u|^{p-4} u_{y}^{2}\right] J}{w}\right\}\left(4_{J}\right) \\
& -\frac{4(p-2)^{2}\left(d^{\prime} c F\right)^{2}|\nabla u|^{p-4} u_{y}^{2}|\nabla u|^{p-4} J}{w} \\
& +\frac{2 d^{\prime} c F(p-2)|\nabla u|^{p-4}|\nabla u|^{q} u_{y}}{w}{ }^{w}{ }^{\prime} F^{2}|\nabla u|^{p-4} u_{y}\left[(p-1)|\nabla u|^{p-2}+(p-2)|\nabla u|^{p-4} u_{y}^{2}\right] \\
& \left.+\frac{2(p-2) c^{3} d^{2} F^{2}}{w}\right\}\left(4_{-}\right) \leq 0 \\
& +\frac{4(p-2)^{2}\left(d^{\prime} c F\right)^{2} c d F|\nabla u|^{p-4} u_{y}^{2}|\nabla u|^{p-4}}{w} \\
& \left.-\frac{2(p-2) d^{\prime} d c^{\prime} c F^{2} u_{y}|\nabla u|^{p-4}\left[|\nabla u|^{p-2}+(p-2)|\nabla u|^{p-4} u_{x}^{2}\right]}{w}\right\}\left(4_{+}\right) \geq 0 \\
& \left.-\frac{2 d^{\prime} c F(p-2)|\nabla u|^{p-4} u_{t} u_{y}}{w}\right\}\left(4_{t}\right) .
\end{aligned}
$$


The other two terms can be rewritten as

$$
\left.\begin{array}{rl}
(5)=2 & \left.c d^{\prime} F(p-2) u_{y}|\nabla u|^{p-6} N \cdot \nabla J-\frac{2(p-2) c d^{\prime} F u_{y}^{3}|\nabla u|^{p-6} M \cdot \nabla J}{w}\right\}\left(5_{\nabla}\right) \\
& -2(p-2) c^{2} d^{\prime} d F^{\prime} F u_{y}|\nabla u|^{p-6}\left[u_{x}^{2}+2 u_{y}^{2}\right] J-4(p-2)\left(c d^{\prime} F\right)^{2} u_{y}^{2}|\nabla u|^{p-6} J \\
& +\frac{2(p-2) c^{2} d^{\prime} d F^{\prime} F u_{y}^{2}|\nabla u|^{p-6} u_{y}\left[(p-1)|\nabla u|^{p-2}+(p-2)|\nabla u|^{p-4} u_{y}^{2}\right] J}{w} \\
& +\frac{4(p-2)^{2}\left(d^{\prime} c F\right)^{2} u_{y}^{4}|\nabla u|^{2 p-10} J}{w} \\
& +(p-2) c d^{\prime} F u_{y}|\nabla u|^{q-2}+2(p-2) c^{3} d^{\prime} d^{2} F^{\prime} F^{2} u_{y}|\nabla u|^{p-6}\left[u_{x}^{2}+2 u_{y}^{2}\right] \\
& +\frac{2(p-2) c^{\prime} c d^{\prime} d F^{2} u_{y}|\nabla u|^{p-6} u_{y}^{2}\left[|\nabla u|^{p-2}+(p-2)|\nabla u|^{p-4} u_{x}^{2}\right]}{w} \\
& -\frac{4(p-2)^{2}\left(c d^{\prime} F\right)^{2} c d F u_{y}^{4}|\nabla u|^{2 p-10}}{w} \\
& -2(p-2) c^{\prime} c d^{\prime} d F^{2} u_{y}|\nabla u|^{p-6} u_{x}^{2}+4(p-2)\left(c d^{\prime} F\right)^{2} c d F u_{y}^{2}|\nabla u|^{p-6} \\
& -\frac{\left.2(p-2) c d^{\prime} F u_{y}|\nabla u|^{p-4}\left|u_{y}^{2}\right| \nabla u\right|^{q-2}}{w} \\
& -\frac{2(p-2) c^{3} d^{\prime} d^{2} F^{\prime} F^{2} u_{y}|\nabla u|^{p-6} u_{y}^{2}\left[(p-1)|\nabla u|^{p-2}+(p-2)|\nabla u|^{p-4} u_{y}^{2}\right]}{w} \\
& \left.-\frac{(p-2) c d^{\prime} F u_{y} u_{t}}{|\nabla u|^{2}}+\frac{2(p-2) c d^{\prime} F u_{y}^{2}|\nabla u|^{p-6} u_{t} u_{y}}{w}\right\}\left(5_{t}\right)
\end{array}\right\}\left(5_{+}\right) \leq 0
$$

and (noticing that (6) can be obtained from (5) by formally multiplying with $\frac{-c^{\prime} d^{2} F}{d^{\prime} u_{y}}$ )

$\left.(6)=-2(p-2) c^{\prime} c d^{2} F^{2}|\nabla u|^{p-6} N \cdot \nabla J+\frac{2(p-2) c^{\prime} c d^{2} F^{2} u_{y}^{2}|\nabla u|^{p-6} M \cdot \nabla J}{w}\right\}\left(6_{\nabla}\right)$

$$
\left.\begin{array}{l}
+2(p-2) c^{\prime} c^{2} d^{3} F^{\prime} F^{2}|\nabla u|^{p-6}\left[u_{x}^{2}+2 u_{y}^{2}\right] J+4(p-2) c^{\prime} c^{2} d^{\prime} d^{2} F^{3} u_{y}|\nabla u|^{p-6} J \\
-\frac{2(p-2) c^{\prime} c^{2} d^{3} F^{\prime} F^{2} u_{y}^{2}|\nabla u|^{p-6}\left[(p-1)|\nabla u|^{p-2}+(p-2)|\nabla u|^{p-4} u_{y}^{2}\right] J}{w} \\
-\frac{4(p-2)^{2} c^{\prime} c^{2} d^{\prime} d^{2} F^{3}|\nabla u|^{p-6} u_{y}^{3}|\nabla u|^{p-4} J}{w}
\end{array}\right\}\left(6_{J}\right)
$$


We shall now collect and relabel the numerous positive and negative terms that we just obtained, when expanding (1)-(7) in the process of eliminating $u_{y y}$. A number of positive and negative terms will then be paired together according to certain cancellations. Then, the remaining positive terms, as well as the terms involving $u_{t}$, will be eventually controlled by using the negative terms.

Using that $d^{\prime} \leq 0$ and $F^{\prime}, F^{\prime \prime}, u_{y} \geq 0$, we first have positive terms:

$$
\begin{aligned}
(a):= & -2(p-2) c^{3} d^{\prime} d^{2} F^{\prime} F^{2}|\nabla u|^{p-4} u_{y} \\
(b):= & -\frac{2(p-2) c d^{\prime} F u_{y}^{2}|\nabla u|^{p-6}|\nabla u|^{q} u_{y}}{w} \\
(c):= & -\frac{2(p-2) c^{3} d^{\prime} d^{2} F^{\prime} F^{2} u_{y}|\nabla u|^{p-6} u_{y}^{2}\left[(p-1)|\nabla u|^{p-2}+(p-2)|\nabla u|^{p-4} u_{y}^{2}\right]}{w} \\
(d):= & +2(p-2)\left(c^{\prime}\right)^{2} c d^{3} F^{3}|\nabla u|^{p-6} u_{x}^{2} \\
(e):= & +\frac{2(p-2) c^{\prime} c^{3} d^{4} F^{\prime} F^{3}|\nabla u|^{p-6} u_{y}^{2}\left[(p-1)|\nabla u|^{p-2}+(p-2)|\nabla u|^{p-4} u_{y}^{2}\right]}{w} \\
(f):= & \left.-\frac{2(p-2) d^{\prime} d c^{\prime} c F^{2} u_{y}|\nabla u|^{p-4}\left[|\nabla u|^{p-2}+(p-2)|\nabla u|^{p-4} u_{x}^{2}\right]}{w}\right\}\left(f_{1}\right) \\
& \left.-2(p-2) c^{\prime} c d^{\prime} d F^{2} u_{y}|\nabla u|^{p-6} u_{x}^{2}\right\}\left(f_{2}\right) \\
(g):= & -4(p-2) c^{\prime} c^{3} d^{\prime} d^{3} F^{4} u_{y}|\nabla u|^{p-6} \\
(h):= & +\underbrace{4(p-2)^{2}\left(d^{\prime} c F\right)^{2} c d F|\nabla u|^{p-4} u_{y}^{2}|\nabla u|^{p-4}}_{\left(j_{2}\right)}+\underbrace{4(p-2)\left(c d^{\prime} F\right)^{2} c d F u_{y}^{2}|\nabla u|^{p-6}}_{\left(h_{1}\right)} \\
(i):= & -q d^{\prime} c F|\nabla u|^{q-2} u_{y} \\
(j):= & +q c^{\prime} c d^{2} F^{2}|\nabla u|^{q-2}+\underbrace{2(p-2) c^{\prime} c d^{2} F^{2}|\nabla u|^{p-4} u_{y}^{2}|\nabla u|^{q-2}}_{\left(j_{1}\right)} \\
(l):= & -2(p-1) c d^{\prime} F^{\prime}|\nabla u|^{p-2} u_{y} \\
(m):= & +(4 p-6) c c^{\prime} d^{2} F^{\prime} F|\nabla u|^{p-2} .
\end{aligned}
$$

They give rise to the following decompositions:

$$
\left\{\begin{aligned}
(2) & =(m)+(l)+(a) \\
\left(4_{+}\right) & =\left(f_{1}\right)+\left(h_{1}\right) \\
\left(5_{+}\right) & =(b)+(c)+\left(h_{2}\right)+\left(f_{2}\right) \\
\left(6_{+}\right) & =(d)+(e)+(g)+\left(j_{2}\right) \\
\left(7_{+}\right) & =(i)+\left(j_{1}\right) .
\end{aligned}\right.
$$


We next have terms with a negative sign:

$$
\begin{aligned}
& (\tilde{a}):=+2(p-2) c^{3} d^{\prime} d^{2} F^{\prime} F^{2} u_{y}|\nabla u|^{p-6}\left[u_{x}^{2}+2 u_{y}^{2}\right] \\
& (\tilde{b}):=+\frac{2 d^{\prime} c F(p-2)|\nabla u|^{p-4}|\nabla u|^{q} u_{y}}{w} \\
& (\tilde{c}):=+\frac{2(p-2) c^{3} d^{\prime} d^{2} F^{\prime} F^{2}|\nabla u|^{p-4} u_{y}\left[(p-1)|\nabla u|^{p-2}+(p-2)|\nabla u|^{p-4} u_{y}^{2}\right]}{w} \\
& (\tilde{d}):=-2(p-2) c d F|\nabla u|^{p-4}\left(c^{\prime} d F\right)^{2} \\
& (\tilde{e}):=-2(p-2) c^{\prime} c^{3} d^{4} F^{\prime} F^{3}|\nabla u|^{p-6}\left[u_{x}^{2}+2 u_{y}^{2}\right] \\
& \left.(\tilde{f}):=\frac{2(p-2) c^{\prime} c d^{\prime} d F^{2} u_{y}|\nabla u|^{p-6} u_{y}^{2}\left[|\nabla u|^{p-2}+(p-2)|\nabla u|^{p-4} u_{x}^{2}\right]}{w}\right\}\left(\tilde{f}_{1}\right) \\
& \left.+4(p-2) c^{\prime} c d^{\prime} d F^{2}|\nabla u|^{p-4} u_{y}\right\}\left(\tilde{f}_{2}\right) \\
& (\tilde{g}):=+(p-2) d^{\prime} c F|\nabla u|^{q-2} u_{y} \\
& (\tilde{h}):=\underbrace{-(p-1) c d F^{\prime \prime}|\nabla u|^{p}}_{\left(\tilde{h}_{1}\right)} \underbrace{+(p-1-q)|\nabla u|^{q} c d F^{\prime}}_{\left(\tilde{h}_{2}\right)} \underbrace{-(p-1) c d^{\prime \prime} F|\nabla u|^{p-4} u_{y}^{2}}_{\left(\tilde{h}_{3}\right)} \\
& (\tilde{i}):=\underbrace{-c d^{\prime \prime} F|\nabla u|^{p-4} u_{x}^{2}}_{\left(\tilde{i}_{1}\right)} \underbrace{-(p-2) c^{\prime} c d^{2} F^{2}|\nabla u|^{q-2}}_{\left(\tilde{i}_{1}\right)} \underbrace{+\frac{4(p-2)^{2} c^{\prime} c^{3} d^{\prime} d^{3} F^{4} u_{y}^{3}|\nabla u|^{2 p-10}}{w}}_{\left(\tilde{i}_{3}\right)} \\
& (\tilde{j}):=\underbrace{-2(p-2)\left(c d^{\prime} F\right)^{2} c d F|\nabla u|^{p-4}}_{\left(\tilde{j}_{1}\right)} \underbrace{-\frac{4(p-2)^{2}\left(c d^{\prime} F\right)^{2} c d F u_{y}^{4}|\nabla u|^{2 p-10}}{w}}_{\left(\tilde{j}_{2}\right)} \\
& (\tilde{l}):=-\frac{2(p-2)\left(c^{\prime}\right)^{2} c d^{3} F^{3}|\nabla u|^{p-6} u_{y}^{2}\left[|\nabla u|^{p-2}+(p-2)|\nabla u|^{p-4} u_{x}^{2}\right]}{w} .
\end{aligned}
$$

With these terms, we have the following decompositions (using $c^{\prime \prime}=0$ ):

$$
\left\{\begin{array}{l}
(1)=(\tilde{d})+\left(\tilde{f}_{2}\right)+\left(\tilde{h}_{1}\right)+\left(\tilde{h}_{3}\right)+\left(\tilde{i}_{1}\right)+\left(\tilde{j}_{1}\right) \\
\left(4_{-}\right)=(\tilde{b})+(\tilde{c}) \\
\left(5_{-}\right)=(\tilde{a})+(\tilde{g})+\left(\tilde{j}_{2}\right)+\left(\tilde{f}_{1}\right) \\
\left(6_{-}\right)=(\tilde{e})+\left(\tilde{i}_{2}\right)+\left(\tilde{i}_{3}\right)+(\tilde{l}) \\
\left(7_{-}\right)=\left(\tilde{h}_{2}\right)-\left(0_{q}\right)-\left(3_{q}\right) .
\end{array}\right.
$$


It follows from (9.4) in Lemma 9.1 and (9.5)-(9.7) that

$$
\begin{aligned}
\mathcal{L} J= & {\left[\left(0_{q}\right)+\left(0_{p}\right)\right] } \\
& +\left[(\tilde{d})+\left(\tilde{f}_{2}\right)+\left(\tilde{h}_{1}\right)+\left(\tilde{h}_{3}\right)+\left(\tilde{i}_{1}\right)+\left(\tilde{j}_{1}\right)\right] \\
& +[(m)+(l)+(a)] \\
& +\left[-\left(0_{p}\right)+\left(3_{t}\right)+\left(3_{q}\right)\right] \\
& +[(\tilde{b})+(\tilde{c})]+\left[\left(f_{1}\right)+\left(h_{1}\right)\right]+\left[\left(4_{\nabla}\right)+\left(4_{J}\right)+\left(4_{t}\right)\right] \\
& +\left[(\tilde{a})+(\tilde{g})+\left(\tilde{j}_{2}\right)+\left(\tilde{f}_{1}\right)\right]+\left[(b)+(c)+\left(h_{2}\right)+\left(f_{2}\right)\right]+\left[\left(5_{\nabla}\right)+\left(5_{J}\right)+\left(5_{t}\right)\right] \\
& +\left[(\tilde{e})+\left(\tilde{i}_{2}\right)+\left(\tilde{i}_{3}\right)+(\tilde{l})\right]+\left[(d)+(e)+(g)+\left(j_{2}\right)\right]+\left[\left(6_{\nabla}\right)+\left(6_{J}\right)+\left(6_{t}\right)\right] \\
& +\left[\left(\tilde{h}_{2}\right)-\left(0_{q}\right)-\left(3_{q}\right)\right]+\left[(i)+\left(j_{1}\right)\right] .
\end{aligned}
$$

Reordering the terms, we obtain

$$
\begin{aligned}
\mathcal{L} J= & (a)+(b)+(c)+(d)+(e)+(f)+(g)+(h)+(i)+(j)+(l)+(m) \\
& +(\tilde{a})+(\tilde{b})+(\tilde{c})+(\tilde{d})+(\tilde{e})+(\tilde{f})+(\tilde{g})+(\tilde{h})+(\tilde{i})+(\tilde{j})+(\tilde{l}) \\
& +\left[\left(3_{t}\right)+\left(4_{t}\right)+\left(5_{t}\right)+\left(6_{t}\right)\right] \\
& +\left[\left(4_{\nabla}\right)+\left(5_{\nabla}\right)+\left(6_{\nabla}\right)\right]+\left[\left(4_{J}\right)+\left(5_{J}\right)+\left(6_{J}\right)\right] .
\end{aligned}
$$

Collecting the terms with $J$ (reps., $\nabla J$ ) in (9.8), together with those in $\mathcal{A}_{2}$ (resp., $\mathcal{H}_{2}$ ) and using (9.3), we define

$$
\begin{aligned}
\mathcal{H}:=\mathcal{H}_{1} & +q|\nabla u|^{q-2} \nabla u+\frac{2(p-2) d^{\prime} c F|\nabla u|^{p-4} u_{y} M}{w} \\
& +2 c d^{\prime} F(p-2) u_{y}|\nabla u|^{p-6} N-\frac{2(p-2) c d^{\prime} F u_{y}^{3}|\nabla u|^{p-6} M}{w} \\
& -2(p-2) c^{\prime} c d^{2} F^{2}|\nabla u|^{p-6} N+\frac{2(p-2) c^{\prime} c d^{2} F^{2} u_{y}^{2}|\nabla u|^{p-6} M}{w}
\end{aligned}
$$

and

$$
\begin{aligned}
\mathcal{A}:=\mathcal{A}_{1} & -q c^{\prime} d F|\nabla u|^{q-2} \\
& -\frac{2(p-2) c^{2} d^{\prime} d F^{\prime} F|\nabla u|^{p-4} u_{y}\left[(p-1)|\nabla u|^{p-2}+(p-2)|\nabla u|^{p-4} u_{y}^{2}\right]}{w} \\
& -\frac{4(p-2)^{2}\left(d^{\prime} c F\right)^{2}|\nabla u|^{p-4} u_{y}^{2}|\nabla u|^{p-4}}{w} \\
& -2(p-2) c^{2} d^{\prime} d F^{\prime} F u_{y}|\nabla u|^{p-6}\left[u_{x}^{2}+2 u_{y}^{2}\right]-4(p-2)\left(c d^{\prime} F\right)^{2} u_{y}^{2}|\nabla u|^{p-6} \\
& +\frac{2(p-2) c^{2} d^{\prime} d F^{\prime} F u_{y}^{2}|\nabla u|^{p-6} u_{y}\left[(p-1)|\nabla u|^{p-2}+(p-2)|\nabla u|^{p-4} u_{y}^{2}\right]}{w} \\
& +\frac{4(p-2)^{2}\left(d^{\prime} c F\right)^{2} u_{y}^{4}|\nabla u|^{2 p-10}}{w} \\
& +2(p-2) c^{\prime} c^{2} d^{3} F^{\prime} F^{2}|\nabla u|^{p-6}\left[u_{x}^{2}+2 u_{y}^{2}\right]+4(p-2) c^{\prime} c^{2} d^{\prime} d^{2} F^{3} u_{y}|\nabla u|^{p-6} \\
& -\frac{2(p-2) c^{\prime} c^{2} d^{3} F^{\prime} F^{2} u_{y}^{2}|\nabla u|^{p-6}\left[(p-1)|\nabla u|^{p-2}+(p-2)|\nabla u|^{p-4} u_{y}^{2}\right]}{w} \\
& -\frac{4(p-2)^{2} c^{\prime} c^{2} d^{\prime} d^{2} F^{3}|\nabla u|^{p-6} u_{y}^{3}|\nabla u|^{p-4}}{w},
\end{aligned}
$$


where we recall that

$$
\begin{aligned}
\mathcal{H}_{1}:= & (p-2)\left[|\nabla u|^{p-4} \Delta u+(p-4)|\nabla u|^{p-6}\left\langle D^{2} u \nabla u, \nabla u\right\rangle\right] \nabla u \\
& -2(p-2) c d^{\prime} F|\nabla u|^{p-4} L \\
& -2(p-2) c^{\prime} d F|\nabla u|^{p-4} \nabla u+2(p-2)|\nabla u|^{p-4}\left(D^{2} u, \nabla u\right),
\end{aligned}
$$

with $L=\left(\begin{array}{c}0 \\ u_{x}\end{array}\right)$, and

$$
\begin{aligned}
\mathcal{A}_{1}:= & -2(p-1) F^{\prime} c^{\prime} d|\nabla u|^{p-2} \\
& -(p-2) F c^{\prime} d\left[|\nabla u|^{p-4} \Delta u+(p-4)|\nabla u|^{p-6}\left\langle D^{2} u \nabla u, \nabla u\right\rangle\right] \\
& +2(p-2)|\nabla u|^{p-4} F^{2}\left[\left(c^{\prime} d\right)^{2}+\left(d^{\prime} c\right)^{2}\right]+2(p-2) d^{\prime} d F^{\prime} F c^{2}|\nabla u|^{p-4} u_{y} \\
& -2(p-2) c^{\prime} d^{\prime} F|\nabla u|^{p-4} u_{y} .
\end{aligned}
$$

Finally observing that

$$
(a)+(\tilde{a}) \leq 0, \quad(b)+(\tilde{b}) \leq 0, \quad(c)+(\tilde{c}) \leq 0, \quad(d)+(\tilde{d}) \leq 0, \quad(e)+(\tilde{e}) \leq 0
$$

and using $(\tilde{f}),(\tilde{i}),(\tilde{j}),(\tilde{l}) \leq 0$, we obtain the following lemma.

Lemma 9.2. Recalling the definition 8.4) of the parabolic operator $\mathcal{P}$ :

$$
\mathcal{P} J:=J_{t}-|\nabla u|^{p-2} \Delta J-(p-2)|\nabla u|^{p-4}\left\langle D^{2} J \nabla u, \nabla u\right\rangle-\mathcal{H} \cdot \nabla J-\mathcal{A}(x, y, t) J,
$$

we have

$$
\begin{aligned}
\mathcal{P} J \leq & (f)+(g)+(h)+(i)+(j)+(l)+(m)+(\tilde{g})+(\tilde{h}) \\
& +\left(3_{t}\right)+\left(4_{t}\right)+\left(5_{t}\right)+\left(6_{t}\right) .
\end{aligned}
$$

Completion of proof of Proposition 8.1. Starting from Lemma 9.2, we shall estimate the remaining positive and $u_{t}$ terms by the key negative terms $(\tilde{g})$ and $(\tilde{h})$, after appropriate choice of the parameters. An essential tool in this step will the Bernstein-type estimates (see [2, Theorem 1.2])

$$
|\nabla u| \leq C_{0} y^{-1 /(q-p+1)} \quad \text { and } \quad u \leq C_{0} y^{(q-p) /(q-p+1)} \quad \text { in }\left[0, x_{1}\right] \times\left(0, L_{2}\right] \times(0, T),
$$

where $C_{0}=C_{0}\left(p, q, \Omega, \mu,\left\|\nabla u_{0}\right\|_{\infty}\right)>0$, and we will use also the lower bound from (1.11):

$$
|\nabla u| \geq u_{y} \geq \delta_{0}=\mu / 2>0 .
$$

First, using $w \geq|\nabla u|^{p-2}$, we get

$$
(f) \leq-2(p-2) p c^{\prime} c d^{\prime} d F^{2} u_{y}|\nabla u|^{p-4} .
$$

Assume $y_{1} \leq 1$. Due to (9.13), we have

$$
d F \leq C_{0}^{\alpha} y^{-\gamma+\alpha(q-p) /(q-p+1)} \leq C_{0}^{\alpha} .
$$


Here we used that $\gamma \leq \alpha-1$ and hence $\alpha(q-p) /(q-p+1)-\gamma \geq 1-\alpha /(q-p+1) \geq 0$. Assume $k_{0}=k_{0}\left(p, q, \Omega, \mu,\left\|\nabla u_{0}\right\|_{\infty}\right)>0$ sufficiently small so that

$$
0<k_{0} \leq \frac{|\nabla u|^{q-p+2}}{4 p d F} \quad \text { and } \quad 0<k_{0}^{3} \leq \frac{|\nabla u|^{q-p+4}}{8 x^{2} F^{3} d^{3}},
$$

which is possible due to (9.14),$x \leq L_{1}$ and (9.15)). We then have

$$
\begin{aligned}
& (f)+(g)+(\tilde{g}) \\
\leq & \underbrace{\frac{p-2}{2} c d^{\prime} F u_{y}}_{\leq 0}[\underbrace{|\nabla u|^{q-2}-8 k^{3} x^{2} F^{3} d^{3}|\nabla u|^{p-6}+|\nabla u|^{q-2}-4 p k|\nabla u|^{p-4} d F}_{\geq 0}] \leq 0 .
\end{aligned}
$$

Next, we have

$$
\begin{aligned}
& (h) \leq 4(p-2)(p-1)\left(c d^{\prime} F\right)^{2} c d F u_{y}^{2}|\nabla u|^{p-6} \\
& (j) \leq(q+2(p-2)) c^{\prime} c d^{2} F^{2}|\nabla u|^{q-2}
\end{aligned}
$$

and, owing to Lemma 3.4 ,

$$
\begin{aligned}
\left(3_{t}\right) & \leq(p-2) c d F^{\prime} K \\
\left(4_{t}\right)+\left(5_{t}\right) & \leq 5(p-2) c\left|d^{\prime}\right| F \frac{\left|u_{t}\right| u_{y}}{|\nabla u|^{2}} \leq 5(p-2) c\left|d^{\prime}\right| F \frac{K u_{y}}{|\nabla u|^{2}} \\
\left(6_{t}\right) & \leq \frac{3(p-2) c^{\prime} c d^{2} F^{2} K}{|\nabla u|^{2}} .
\end{aligned}
$$

Here and in the rest of the proof, $K$ denotes a constant depending on $\left\|u_{0}\right\|_{C^{2}}, p$ and $q$. Consequently

$$
\begin{aligned}
\mathcal{P} J \leq & (h)+(i)+(j)+(l)+(m)+\left(3_{t}\right)+\left(4_{t}\right)+\left(5_{t}\right)+\left(6_{t}\right)+(\tilde{h}) \\
\leq & 4(p-2)(p-1)\left(c d^{\prime} F\right)^{2} c d F u_{y}^{2}|\nabla u|^{p-6}+(q+2(p-2)) c^{\prime} c d^{2} F^{2}|\nabla u|^{q-2} \\
& +5(p-2) c\left|d^{\prime}\right| F \frac{K u_{y}}{|\nabla u|^{2}}+2(p-1) c\left|d^{\prime}\right| F^{\prime}|\nabla u|^{p-2} u_{y} \\
& +(4 p-6) c c^{\prime} d^{2} F^{\prime} F|\nabla u|^{p-2}+q\left|d^{\prime}\right| c F|\nabla u|^{q-2} u_{y} \\
& +\frac{3(p-2) c^{\prime} c d^{2} F^{2} K}{|\nabla u|^{2}}+(p-2) c d F^{\prime} K \\
& -(p-1) c d F^{\prime \prime}|\nabla u|^{p}+(p-1-q)|\nabla u|^{q} c d F^{\prime}-(p-1) c d^{\prime \prime} F|\nabla u|^{p-4} u_{y}^{2}
\end{aligned}
$$

hence

$$
\begin{aligned}
\frac{\mathcal{P} J}{c d F} & \leq-(q-p+1) \alpha \frac{|\nabla u|^{q}}{u}-(p-1) \alpha(\alpha-1) \frac{|\nabla u|^{p}}{u^{2}}-(p-1) \frac{\gamma(\gamma+1)|\nabla u|^{p-4} u_{y}^{2}}{y^{2}} \\
& +\frac{2 \gamma \alpha(p-1)}{y} \frac{|\nabla u|^{p-2} u_{y}}{u}+4(p-2)(p-1) k^{2} \gamma^{2} x^{2} u^{2 \alpha} y^{-2 \gamma} \frac{|\nabla u|^{p-6} u_{y}^{2}}{y^{2}} \\
& +5(p-2) \gamma K|\nabla u|^{-2} \frac{u_{y}}{y}+(4 p-6) k \alpha \frac{u^{\alpha-1}|\nabla u|^{p-2}}{y^{\gamma}}+(q+2(p-2)) k \frac{u^{\alpha}|\nabla u|^{q-2}}{y^{\gamma}} \\
& +q \gamma \frac{|\nabla u|^{q-2} u_{y}}{y}+(p-2) \frac{\alpha K}{u}+\frac{3(p-2) k y^{-\gamma} u^{\alpha} K}{|\nabla u|^{2}} .
\end{aligned}
$$


Using Young's inequality, we obtain that

$$
\frac{2 \gamma \alpha}{y} \frac{|\nabla u|^{p-2} u_{y}}{u} \leq \alpha(\alpha-1) \frac{|\nabla u|^{p}}{u^{2}}+\frac{\alpha \gamma^{2}}{\alpha-1} \frac{|\nabla u|^{p-4} u_{y}^{2}}{y^{2}}
$$

hence

$$
\begin{aligned}
& \frac{2 \gamma \alpha(p-1)}{y} \frac{|\nabla u|^{p-2} u_{y}}{u}-(p-1) \alpha(\alpha-1) \frac{|\nabla u|^{p}}{u^{2}}-(p-1) \frac{\gamma(\gamma+1)|\nabla u|^{p-4} u_{y}^{2}}{y^{2}} \\
& \leq\left(\frac{\alpha \gamma^{2}}{\alpha-1}-\gamma(\gamma+1)\right) \frac{(p-1)|\nabla u|^{p-4} u_{y}^{2}}{y^{2}}=-\frac{2 \gamma \sigma(p-1)|\nabla u|^{p-4} u_{y}^{2}}{y^{2}}
\end{aligned}
$$

By (9.13), we have also

$$
u|\nabla u|^{q-p} \leq C_{0}^{q-p+1} .
$$

Using again Young's inequality, and (9.19), we have

$$
\begin{aligned}
& q \gamma \frac{|\nabla u|^{q-2} u_{y}}{y} \leq \frac{\sigma \gamma|\nabla u|^{p-4} u_{y}^{2}}{2 y^{2}}+\frac{q^{2} \gamma}{2 \sigma}|\nabla u|^{2 q-p} \leq \frac{\sigma \gamma|\nabla u|^{p-4} u_{y}^{2}}{2 y^{2}}+\frac{q^{2} \gamma C_{0}^{q-p+1}}{2 \sigma} \frac{|\nabla u|^{q}}{u}, \\
& 5(p-2) \gamma K|\nabla u|^{-2} \frac{u_{y}}{y} \leq \frac{\sigma \gamma|\nabla u|^{p-4} u_{y}^{2}}{2 y^{2}}+\left[\frac{25(p-2)^{2} \gamma K^{2}|\nabla u|^{-p-q} u}{2 \sigma}\right] \frac{|\nabla u|^{q}}{u} .
\end{aligned}
$$

Next, using (9.13) and (9.14), it follows that

$$
\begin{aligned}
& k \alpha(4 p-6) \frac{u^{\alpha-1}|\nabla u|^{p-2}}{y^{\gamma}} \leq k \alpha(4 p-6) C_{0}^{\alpha-1} y^{(\alpha-1)\left(2 \sigma-\frac{1}{q-p+1}\right)+2} \frac{|\nabla u|^{2}}{u_{y}^{2}} \frac{|\nabla u|^{p-4} u_{y}^{2}}{y^{2}} \\
& \leq k \alpha(4 p-6) \delta_{0}^{-2} C_{0}^{\alpha+1} y^{(\alpha-1)\left(2 \sigma-\frac{1}{q-p+1}\right)+\frac{2(q-p)}{q-p+1}} \frac{|\nabla u|^{p-4} u_{y}^{2}}{y^{2}}, \\
& (q+2(p-2)) k \frac{u^{\alpha}|\nabla u|^{q-2}}{y^{\gamma}} \leq(q+2(p-2)) k C_{0}^{\alpha+q-p} y^{(\alpha-1)\left(2 \sigma-\frac{1}{q-p+1}\right)+2} \frac{|\nabla u|^{2}}{u_{y}^{2}} \frac{|\nabla u|^{p-4} u_{y}^{2}}{y^{2}} \\
& \leq(q+2(p-2)) k \frac{C_{0}^{\alpha+q-p+2}}{\delta_{0}^{2}} y^{(\alpha-1)\left(2 \sigma-\frac{1}{q-p+1}\right)+\frac{2(q-p)}{q-p+1}} \frac{|\nabla u|^{p-4} u_{y}^{2}}{y^{2}}
\end{aligned}
$$

and

$$
k^{2} \gamma^{2} \frac{x^{2} u^{2 \alpha}}{y^{2 \gamma}} \frac{|\nabla u|^{p-6} u_{y}^{2}}{y^{2}} \leq k^{2} \gamma^{2} \frac{x^{2} C_{0}^{2 \alpha} y^{-2 \gamma+2 \alpha(q-p) /(q-p+1)}}{\delta_{0}^{2}} \frac{|\nabla u|^{p-4} u_{y}^{2}}{y^{2}} .
$$

Finally, using that $u \geq \mu y$, we have

$$
\frac{\alpha K}{u}=\frac{\alpha K y^{2}}{u|\nabla u|^{p-4} u_{y}^{2}} \frac{|\nabla u|^{p-4} u_{y}^{2}}{y^{2}} \leq \frac{\alpha K y}{\mu \delta_{0}^{p-2}} \frac{|\nabla u|^{p-4} u_{y}^{2}}{y^{2}} .
$$

Using the bounds $u \leq\left\|u_{0}\right\|_{\infty}$ and (9.14) we have

$$
\frac{k y^{-\gamma} u^{\alpha} K}{|\nabla u|^{2}} \leq \frac{k\left\|u_{0}\right\|_{\infty}^{\alpha} K y^{2-\gamma}}{\delta_{0}^{p}} \frac{|\nabla u|^{p-4} u_{y}^{2}}{y^{2}}
$$


Combining (9.17)-(9.26), we get that

$$
\begin{aligned}
\frac{\mathcal{P} J}{c d F} \leq & \frac{|\nabla u|^{q}}{u}\left(\frac{q^{2} \gamma C_{0}^{q-p+1}}{2 \sigma}+25(p-2)^{2} \gamma \frac{K^{2}\left\|u_{0}\right\|_{\infty}}{2 \sigma \delta_{0}^{q+p}}-\alpha(q-p+1)\right) \\
& +\frac{|\nabla u|^{p-4} u_{y}^{2}}{y^{2}}\left\{k\left((q+2(p-2)) C_{0}^{\alpha+q-p+2} \delta_{0}^{-2}\right) y^{(\alpha-1)\left(2 \sigma-\frac{1}{q-p+1}\right)+\frac{2(q-p)}{q-p+1}}\right. \\
& +k\left(\alpha(4 p-6) \delta_{0}^{-2} C_{0}^{\alpha+1}\right) y^{(\alpha-1)\left(2 \sigma-\frac{1}{q-p+1}\right)+\frac{2(q-p)}{q-p+1}} \\
& +4(p-2)(p-1) k^{2} \gamma^{2} x^{2} C_{0}^{2 \alpha} y^{-2 \gamma+\frac{2 \alpha(q-p)}{q-p+1}} \delta_{0}^{-2} \\
& \left.+(p-2) \frac{\alpha K y}{\mu \delta_{0}^{p-2}}+3(p-2) \frac{k\left\|u_{0}\right\|_{\infty}^{\alpha} K y^{2-\gamma}}{\delta_{0}^{p-2}}-(2 p-3) \sigma \gamma\right\} .
\end{aligned}
$$

Now, we may choose $\alpha=\alpha\left(p, q, \Omega, \mu,\left\|u_{0}\right\|_{C^{2}}, \sigma\right)>1$ close enough to 1 in such a way that $\gamma=(\alpha-1)(1-2 \sigma)$ is small enough to satisfy

$$
\gamma\left[\frac{q^{2}}{2 \sigma} C_{0}^{q-p+1}+25(p-2)^{2} \frac{K^{2}\left\|u_{0}\right\|_{\infty}}{2 \sigma \delta_{0}^{q-p}}\right] \leq(q-p+1) \alpha
$$

and

$$
\begin{aligned}
& (\alpha-1)\left(2 \sigma-\frac{1}{q-p+1}\right)+\frac{2(q-p)}{q-p+1} \geq 0, \\
& \alpha \frac{q-p}{q-p+1}-(\alpha-1)(1-2 \sigma) \geq 0 .
\end{aligned}
$$

Finally, once $\alpha$ is fixed (hence $\gamma$ is also fixed small), recalling that $y \leq y_{1} \leq 1, x \leq L_{1}$ and $\gamma \leq 2$, we take $k_{0}=k_{0}\left(p, q, \Omega, \mu,\left\|u_{0}\right\|_{C^{2}}, \sigma\right)>0$ possibly smaller, in such a way that

$$
\begin{aligned}
& k_{0}\left((q+2(p-2)) C_{0}^{\alpha+q-p+2} \delta_{0}^{-2}+\alpha(4 p-6) \delta_{0}^{-2} C_{0}^{\alpha+1}\right) \\
& +k_{0}^{2}\left(4(p-2)(p-1) \gamma^{2} L_{1}^{2} C_{0}^{2 \alpha} \delta_{0}^{-2}\right)+k_{0} \frac{3(p-2)\left\|u_{0}\right\|_{\infty}^{\alpha} K}{\delta_{0}^{p-2}} \leq \frac{2 p-3}{2} \sigma \gamma
\end{aligned}
$$

and next we take $y_{1}=y_{1}\left(p, q, \Omega, \mu,\left\|u_{0}\right\|_{C^{2}}, \sigma\right)>0$ small enough such that

$$
\frac{(p-2) \alpha K y_{1}}{\mu \delta_{0}^{p-2}} \leq \frac{2 p-3}{2} \sigma \gamma
$$

Then it follows from (9.27) that

$$
\mathcal{P} J \leq 0 \quad \text { in } D \times(T / 2, T)
$$

Finally, we need to check (8.6). The continuity statement is clear from the definition of $\mathcal{A}, \mathcal{H}$. Let us show that $\mathcal{A}$ is bounded in $D \times(T / 2, \tau)$ for each $\tau<T$. For this purpose, let us observe that due to $|\nabla u| \leq C(\tau), u \leq C(\tau) y$ and $\alpha-1 \geq \gamma$, we have for $y \leq 1$ and $\tau \in(T / 2, T)$

$$
\begin{array}{r}
\left|F^{\prime} d\right|=\alpha u^{\alpha-1} y^{-\gamma} \leq C^{\alpha-1}(\tau) y^{\alpha-1-\gamma} \leq \alpha C^{\alpha-1}(\tau) \\
\left|F d^{\prime}\right|=\gamma u^{\alpha} y^{-\gamma-1} \leq \gamma C^{\alpha}(\tau) \\
|F d|=u^{\alpha} y^{-\gamma} \leq C^{\alpha}(\tau) .
\end{array}
$$


We also have by (9.13) and (9.14):

$$
|\nabla u|^{r} \leq \begin{cases}C^{r}(\tau), & \text { if } r>0, \\ \delta_{0}^{r}, & \text { if } r<0 .\end{cases}
$$

The assertion then follows easily from (9.10), (9.12) and (2.7).

\section{APPENDIX 1. Proof of regularity results (The- orem 2.2)}

Proof of Theorem 2.2(i). We assume $\delta_{0}:=\inf _{Q_{T}}|\nabla u|>0$. Fix $0<\tau<T$ and let $M_{\tau}=\|\nabla u\|_{L^{\infty}\left(Q_{\tau}\right)}<\infty$. We pick smooth functions $b=b_{\tau}$ and $F=F_{\tau}$ with the following properties:

$$
\begin{aligned}
& b(s)=s^{(p-2) / 2} \text { and } F(s)=s^{q / 2} \quad \text { for } \delta_{0}^{2} \leq s \leq M_{\tau}^{2}, \\
& \inf _{[0, \infty)} b>0, \quad b^{\prime} \geq 0, \quad b^{\prime}(s)=0 \text { for } s \text { large enough, } \quad F \geq 0, \quad \sup _{[0, \infty)} F<\infty .
\end{aligned}
$$

By the results in [13, Chapter V] (see Remark 10.1 below for details), there exists a (unique) classical solution $v=v_{\tau} \in C^{2+\alpha, 1+\alpha / 2}(\bar{\Omega} \times(0, \tau)) \cap C\left(\bar{Q}_{\tau}\right)$, for some $\alpha \in(0,1)$, of the problem

$$
\begin{aligned}
v_{t}-\nabla \cdot\left(b\left(|\nabla v|^{2}\right) \nabla v\right) & =F\left(|\nabla v|^{2}\right) \quad \text { in } Q_{\tau} \\
v & =g \text { on } S_{\tau} \\
v(\cdot, 0) & =u_{0} \quad \text { in } \Omega .
\end{aligned}
$$

Since $v$ is also a weak solution of (2.1)-(2.3) in $Q_{\tau}$, by uniqueness of weak solutions (cf. Theorem 2.1(ii)), it follows that $u=v_{\tau}$ on $Q_{\tau}$, hence (2.7).

Remark 10.1. More precisely, in the special case when $u_{0} \in C^{2+\alpha}(\bar{\Omega})$ and $u_{0}$ satisfies the second order compatibility conditions, the existence of $v$ claimed in the above proof follows from [13, Theorem V.6.1]. In the general case $u_{0} \in W^{1, \infty}(\Omega)$, with $u_{0}=g$ on $\partial \Omega$, this follows by a standard approximation procedure of $u_{0}$ by such smooth $u_{0, n}$. Namely, if $v_{n}$ denotes the solution originating from $u_{0, n}$, then, by [13, Theorems V.4.1, V.1.1 and $V .5 .4]$ respectively, we get uniform a priori estimates for the sequence $v_{n}$ in the spaces $L^{\infty}\left(0, \tau ; W^{1, \infty}(\Omega)\right), C^{\alpha}\left(\bar{Q}_{\tau}\right)$ and $C_{l o c}^{2+\alpha, 1+\alpha / 2}(\bar{\Omega} \times(0, \tau])$ for some $\alpha \in(0,1)$. We may then pass to the limit along a subsequence and obtain a solution with the announced properties.

In the proof of Theorem 2.2(ii)(iii), we shall use the following local regularity lemma. We note that only statement (ii) will be used here. The global version of statement (i) was already proved in Theorem 2.2(i). However, we give and prove its local version for completeness, since it was mentioned without proof in [2, p. 2487].

Lemma 10.1. Under the assumptions of Theorem 2.1, let $u$ be the (maximal) weak solution of (1.1) and let $P_{0}=\left(x_{0}, y_{0}, t_{0}\right) \in Q_{T}$. Assume $\left|\nabla u\left(P_{0}\right)\right|>0$. Then:

(i) for some $\alpha \in(0,1)$, $u$ is a classical $C^{2+\alpha, 1+\alpha / 2}$-solution on a space-time neighborhood of $P_{0}$;

(ii) for some $\beta \in(0,1), \nabla u$ is $C^{2+\beta, 1+\beta / 2}$ on a space-time neighborhood of $P_{0}$. 
Proof. (i) Since, by Theorem 2.1(iii), $\nabla u$ is continuous in $Q_{T}$, there exist $\lambda, \rho, M_{2}>0$ such that

$$
\lambda \leq|\nabla u| \leq M_{2} \quad \text { in } Q^{\rho}:=B_{\rho}\left(x_{0}, y_{0}\right) \times\left[t_{0}-\rho, t_{0}+\rho\right] \subset Q_{T} .
$$

For any unit vector $\vec{e}$ and $0<h<\rho / 2$, let us introduce the differential quotients

$$
D_{h} u=h^{-1}\left(\tau_{h} u-u\right), \quad \text { where } \tau_{h} u=u((x, y)+h \vec{e}, t) .
$$

We have

$$
\left|\nabla \tau_{h} u\right|^{q}-|\nabla u|^{q}=d^{h}(x, y, t) \cdot \nabla\left(\tau_{h} u-u\right) \text { in } Q^{\rho / 2},
$$

where $\left|d^{h}(x, y, t)\right| \leq C$ independent of $h$. Next denote $b(s)=s^{(p-2) / 2}$ and $a_{i}(p)=b\left(|p|^{2}\right) p_{i}$ where $p=\left(p_{1}, p_{2}\right)$, so that $\Delta_{p} u=\partial_{i}\left(a_{i}(\nabla u)\right)$. Following [13, p.445], we write

$$
\left.a_{i}\left(\nabla \tau_{h} u\right)-a_{i}(\nabla u)=\int_{0}^{1} \frac{d}{d s} a_{i}\left(s \nabla \tau_{h} u+(1-s) \nabla u\right)\right) d s=\tilde{a}_{i j}^{h} \partial_{j}\left(\tau_{h} u-u\right),
$$

where

$$
\left.\tilde{a}_{i j}^{h}(x, y, t)=\int_{0}^{1} \frac{\partial a_{i}}{\partial p_{j}}\left(s \nabla \tau_{h} u+(1-s) \nabla u\right)\right) d s .
$$

Subtracting the PDE in (1.1) for $u$ and for $\tau_{h} u$ and dividing by $h$, we see that $D_{h} u$ is a local weak solution of

$$
\partial_{t}\left(D_{h} u\right)-\partial_{i}\left[\tilde{a}_{i j}^{h} \partial_{j}\left(D_{h} u\right)\right]=d^{h}(x, y, t) \cdot \nabla\left(D_{h} u\right) \quad \text { in } Q^{\rho / 2} .
$$

Moreover, since $\frac{\partial a_{i}}{\partial p_{j}} \xi_{i} \xi_{j}=b\left(|p|^{2}\right)|\xi|^{2}+2 b^{\prime}\left(|p|^{2}\right) p_{i} p_{j} \xi_{i} \xi_{j} \geq b\left(|p|^{2}\right)|\xi|^{2} \geq \lambda^{p-2}|\xi|^{2}$ in $Q^{\rho / 2}$ by (10.1), we have

$$
\tilde{a}_{i j}^{h} \xi_{i} \xi_{j} \geq \lambda^{p-2}|\xi|^{2} \quad \text { in } Q^{\rho / 2}
$$

We then test (10.2) with $\varphi^{2} D_{h} u$, where $\varphi \in C_{0}^{\infty}\left(Q^{\rho / 2}\right)$ is a cut-off function such that $\varphi=1$ on $Q^{\rho / 3}$. By integration by parts and some simple manipulations, it is easy to see that

$$
\lambda^{p-2} \int_{Q^{\rho / 3}}\left|\nabla D_{h} u\right|^{2} d x d y d t \leq \int_{Q^{\rho / 2}} \tilde{a}_{i j}^{h} \partial_{i}\left(D_{h} u\right) \partial_{j}\left(D_{h} u\right) \varphi^{2} d x d y d t \leq C .
$$

It follows that $D^{2} u \in L^{2}\left(Q^{\rho / 3}\right)$. Consequently, we obtain that $u \in W_{2}^{2,1}\left(Q^{\rho / 3}\right)$ and is a local strong solution of equation (1.1) written in nondivergence form, i.e.:

$$
u_{t}-a_{i j} u_{i j}=f \text { in } Q^{\rho / 3}, \quad \text { where } a_{i j}=|\nabla u|^{p-2}\left[\delta_{i j}+(p-2) \frac{u_{i} u_{j}}{|\nabla u|^{2}}\right], \quad f=|\nabla u|^{q} .
$$

Since, by Theorem 2.1(iii), $a_{i j}, f$ are Hölder continuous in $\bar{Q}^{\rho / 3}$, it follows from interior Schauder parabolic regularity [13, Theorem III.12.2] that, for some $\alpha \in(0,1)$,

$$
u \in C^{2+\alpha, 1+\alpha / 2}\left(\bar{Q}^{\rho / 4}\right) .
$$

(ii) Thanks to (10.4), we know that $u$ is a classical solution of (10.3) in $Q^{\rho / 4}$. Keeping the above notation, for $0<h<\rho / 8$, we then have

$$
\left(D_{h} u\right)_{t}-a_{i j}\left(D_{h} u\right)_{i j}=F_{h}:=D_{h} f+\left(D_{h} a_{i j}\right)\left(\tau_{h} u_{i j}\right) \quad \text { in } Q^{\rho / 8} .
$$


Moreover, as a consequence of (10.4), we have, for $1<r<\infty$,

$$
\left\|F_{h}\right\|_{L^{r}\left(Q^{\rho / 8}\right)} \leq C\|\nabla f\|_{L^{r}\left(Q^{\rho / 4}\right)}+\|\nabla A\|_{L^{r}\left(Q^{\rho / 4}\right)}\left\|D^{2} u\right\|_{L^{\infty}\left(Q^{\rho / 4}\right)} \leq C, \quad 0<h<\rho / 8 .
$$

It thus follows from interior parabolic $L^{r}$ estimates (see [13, Theorem III.12.2]) that, for $0<h<\rho / 8$,

$$
\left\|D^{2} D_{h} u\right\|_{L^{r}\left(Q^{\rho / 16}\right)}+\left\|\partial_{t} D_{h}\right\|_{L^{r}\left(Q^{\rho / 16}\right)} \leq C(\rho)\left(\left\|F_{h}\right\|_{L^{r}\left(Q^{\rho / 8}\right)}+\left\|D_{h} u\right\|_{L^{r}\left(Q^{\rho / 8}\right)}\right) \leq C .
$$

We deduce that $D u_{t}, D^{3} u \in L_{l o c}^{r}\left(Q_{T}\right)$. Then differentiating (10.3) in space, we see that the function $z=\partial_{\ell} u_{x}(\ell=1,2)$ is a local strong solution of

$$
z_{t}-a_{i j} z_{i j}=\tilde{f} \quad \text { in } Q^{\rho / 16}
$$

where $\tilde{f}=\partial_{\ell} f-u_{i j} \partial_{\ell} a_{i j}$. Since $a_{i j}, \tilde{f}$ are Hölder continuous in $\bar{Q}^{\rho / 16}$ due to (10.4), it follows from interior Schauder parabolic regularity [13, Theorem III.12.2] that $z \in$ $C^{2+\alpha, 1+\alpha / 2}\left(\bar{Q}^{\rho / 20}\right)$ for some $\alpha \in(0,1)$.

Proof of Theorem 2.2 (continued). (ii) This is a direct consequence of Lemma 10.1,

(iii) It follows from (i)(ii) that $v=u_{x} \in C^{2,1}\left(Q_{T}\right) \cap C(\bar{\Omega} \times(0, T))$ is a classical solution of (10.5) in $Q_{T}$, where $a_{i j}$ are defined in (10.3). Moreover, $v=g_{x}=0$ on $S_{T}$. Taking $\theta(t)$ a cut-off in time and setting $w=\theta v$, we see that $w$ solves

$$
w_{t}-a_{i j} w_{i j}=\bar{f}:=\theta \tilde{f}+\theta_{t} v \quad \text { in } Q_{T},
$$

with 0 initial-boundary conditions. By [18, Theorem 4.28], since $\bar{f}$ is locally Hölder continuous in $\bar{\Omega} \times[0, T)$ due to (i), there exists a solution to this problem in $C^{2+\beta, 1+\beta / 2}(\bar{\Omega} \times$ $[0, T))$ for some $\beta \in(0,1)$. Since we have uniqueness in the class $C^{2,1}\left(Q_{T}\right) \cap C(\bar{\Omega} \times[0, T))$ by the maximum principle, the conclusion (2.9) follows.

\section{APPENDIX 2. A parabolic version of Serrin's corner lemma}

In [17, p. 512], a Serrin corner property in a rectangle was shown for a parabolic equation involving the Laplacian. This was proved by comparison with a suitable product of functions of $x, t$ and $y, t$. This result and method are no longer sufficient here and we shall establish a result for general nondivergence operators by modifying the original proof of [22] for the elliptic case.

Proposition 11.1. Let $0<X_{1}<\hat{X}_{1}, 0<Y_{1}<\hat{Y}_{1}, \hat{D}=\left(0, \hat{X}_{1}\right) \times\left(0, \hat{Y}_{1}\right) \subset \mathbb{R}^{2}$, $0<\tau_{1}<\tau_{2}, \hat{D}_{\tau}=\hat{D} \times\left(\tau_{1}, \tau_{2}\right)$. Let the coefficients $a_{i j}=a_{i j}(x, y, t)$ satisfy

$$
a_{i j} \xi_{i} \xi_{j} \geq \lambda|\xi|^{2} \quad \text { in } \overline{\hat{D}_{\tau}}
$$

for some $\lambda>0$ and assume that

$$
a_{i j}, B_{i} \in C\left(\overline{\hat{D}_{\tau}}\right), \quad a_{12}+a_{21} \geq-C(x \wedge y), \quad B_{1} \geq-C x, \quad B_{2} \geq-C y \quad \text { in } \overline{\hat{D}_{\tau}} .
$$


Let $z \in C^{2,1}\left(\hat{D}_{\tau}\right) \cap C\left(\overline{\hat{D}_{\tau}}\right)$ satisfy

$$
\mathcal{L} z:=z_{t}-a_{i j} z_{i j}-B_{i} z_{i} \leq 0 \quad \text { in } \hat{D}_{\tau}, \quad z(x, y, t) \leq 0 \text { in }{\hat{D_{\tau}}}_{\tau}, \quad z(0,0, t)=0 .
$$

Then, for each $t_{0} \in\left(\tau_{1}, \tau_{2}\right)$, there exists $c_{0}>0$ such that

$$
z \leq-c_{0} x y \quad \text { in }\left(0, X_{1}\right) \times\left(0, Y_{1}\right) \times\left[t_{0}, \tau_{2}\right) .
$$

Proof. Let $a=\min \left(X_{1}, Y_{1}, \frac{t_{0}-\tau_{1}}{2}\right)$ and $\tau_{3}=\frac{\tau_{1}+t_{0}}{2}$, so that $\tau_{1}<\tau_{3}<t_{0}<\tau_{2}$. Fix $t_{1} \in\left[t_{0}, \tau_{2}\right)$ and let

$$
K_{1}:=\left\{(x, y, t) ; x^{2}+(a-y)^{2}+\left(t_{1}-t\right)^{2}<a^{2}, x>0, t \leq t_{1}\right\} .
$$

Observe that $K_{1} \subset \hat{D} \times\left[\tau_{3}, t_{1}\right]$ and set $K_{2}=B((0,0), a / 2) \times\left[\tau_{3}, t_{1}\right]$ and $K=K_{1} \cap K_{2}$.

Now set

$$
\bar{v}(x, y, t):=e^{-\alpha\left(x^{2}+(y-a)^{2}+\left(t-t_{1}\right)^{2}\right)}-e^{-\alpha a^{2}}, \quad v(x, y, t)=e^{-\alpha\left(x^{2}+(y-a)^{2}+\left(t-t_{1}\right)^{2}\right)},
$$

with $\alpha>0$ to be chosen later on, and define the auxiliary function $h=x \bar{v}$. It is clear that $h>0$ in $K$. We compute

$$
\begin{gathered}
h_{t}=-2 \alpha x\left(t-t_{1}\right) v, \quad \nabla h=\left(\begin{array}{c}
\bar{v}-2 \alpha x^{2} v \\
-2 \alpha x(y-a) v
\end{array}\right), \\
D^{2} h=v\left(\begin{array}{cc}
-6 \alpha x+4 \alpha^{2} x^{3} & -2 \alpha(y-a)+4 \alpha^{2} x^{2}(y-a) \\
-2 \alpha(y-a)+4 \alpha^{2} x^{2}(y-a) & -2 \alpha x+4 \alpha^{2} x(y-a)^{2}
\end{array}\right), \\
B(x, y, t) \cdot \nabla h=-2 \alpha x v\left[x B_{1}+(y-a) B_{2}\right]+B_{1} \bar{v} .
\end{gathered}
$$

Using that $a_{i j} \xi_{i} \xi_{j} \geq \lambda|\xi|^{2}$, we have

$$
\begin{aligned}
a_{i j} h_{i j}=v a_{11}\left(-6 \alpha x+4 \alpha^{2} x^{3}\right)+v a_{22}\left(-2 \alpha x+4 \alpha^{2} x(y-a)^{2}\right) \\
+v\left(a_{12}+a_{21}\right)\left(-2 \alpha(y-a)+4 x^{2} \alpha^{2}(y-a)\right) \\
=\alpha x v\left[4 \alpha\left(a_{11} x^{2}+(y-a) x\left(a_{12}+a_{21}\right)+(y-a)^{2} a_{22}\right)\right. \\
\left.\quad-6 a_{11}-2 a_{22}-\frac{2(y-a)\left(a_{12}+a_{21}\right)}{x}\right] \\
\geq \alpha x v\left[4 \alpha \lambda\left(x^{2}+(y-a)^{2}\right)-6 a_{11}-2 a_{22}+\frac{2(a-y)\left(a_{12}+a_{21}\right)}{x}\right],
\end{aligned}
$$

hence

$$
\begin{aligned}
\mathcal{L} h \leq & \alpha x v\left[-4 \alpha \lambda\left(x^{2}+(y-a)^{2}\right)+2\left(t_{1}-t\right)+6 a_{11}+2 a_{22}-\frac{2(a-y)\left(a_{12}+a_{21}\right)}{x}\right. \\
& \left.+2 x B_{1}+2(y-a) B_{2}-\frac{B_{1}}{\alpha x}\left(1-e^{\alpha\left(x^{2}+(y-a)^{2}+\left(t-t_{1}\right)^{2}-a^{2}\right)}\right)\right] .
\end{aligned}
$$


On the one hand, on $K$, we have $y<a / 2$, hence $x^{2}+(y-a)^{2}>a^{2} / 4$. On the other hand, using part of assumptions (11.2) along with $0 \leq a-y \leq a$ and $0 \leq$ $1-e^{\alpha\left(x^{2}+(y-a)^{2}+\left(t-t_{1}\right)^{2}-a^{2}\right)} \leq 1$ on $K$, it follows that for $\alpha>1$ large enough,

$$
\begin{aligned}
\mathcal{L} h & \leq \alpha x v\left[-\alpha \lambda a^{2}+2\left(t_{1}-t\right)+6 a_{11}+2 a_{22}+2 C a+2 x B_{1}+2(y-a) B_{2}+C\right] \\
& \leq-\frac{\lambda \alpha^{2} a^{2} x v}{2}<0 \quad \text { in } K .
\end{aligned}
$$

We now set $w=z+\varepsilon h$ where $\varepsilon$ is a positive constant to be chosen. By (11.3) and (11.5), we have

$$
\mathcal{L} w<0 \quad \text { in } K .
$$

Denote $M=\max _{\bar{K}} w \geq 0$. Since $\mathcal{L}$ is (uniformly) parabolic, by the usual proof of the maximum principle, it follows from (11.6) that $w$ cannot attain the value $M$ in $K$ (observe that for each $s \in\left[\tau_{3}, t_{1}\right]$, the section $K \cap\{t=s\}$ is an open, possibly empty, subset of $\mathbb{R}^{2}$ ). To show $M=0$ (for sufficiently small $\varepsilon>0$ ), it thus suffices to verify that $w \leq 0$ on $\partial_{P} K=\partial K \backslash\left(K \cap\left\{t=t_{1}\right\}\right)$. We have $\partial_{P} K=\Gamma_{1} \cup \Gamma_{2}$, where $\Gamma_{1}=\partial K_{1} \cap \bar{K}_{2}$ and $\Gamma_{2}=\partial K_{2} \cap K_{1}$.

On $\Gamma_{1}$ we have either

$$
x^{2}+(y-a)^{2}+\left(t_{1}-t\right)^{2}=a^{2} \quad \text { or } \quad x=0,
$$

so that $h=0$ and $z \leq 0$, hence $w \leq 0$. Next observe that on $\Gamma_{2}$ we have

$$
x^{2}+(y-a)^{2}+\left(t_{1}-t\right)^{2}<a^{2} \text { and } x^{2}+y^{2}=a^{2} / 4,
$$

hence $\tau_{1}<\tau_{3} \leq t \leq t_{1}$ and $a / 8<y<a / 2$ (in other words, $(x, y)$ is "far" from the corners of $\hat{D})$. Therefore, by the Hopf boundary point lemma [19, Theorem 6 p. 174] and the strong maximum principle, there exists $c>0$ (independent of $t_{1}$ ), such that $z \leq-c x$ on $\Gamma_{2}$. Choosing $\varepsilon \in(0, c)$, we then have $w \leq-c x+\varepsilon x<0$ on $\Gamma_{2}$.

We have thus proved that $M=0$ that is, $w \leq 0$ in $K$. Letting $\tilde{a}:=a /(2 \sqrt{2})$ and noting that $\{0<x \leq y<\tilde{a}\} \times\left\{t_{1}\right\} \subset K$, we get

$$
\begin{aligned}
z\left(x, y, t_{1}\right) & \leq-\varepsilon h\left(x, y, t_{1}\right)=-\varepsilon x e^{-\alpha a^{2}}\left(e^{\alpha\left(a^{2}-x^{2}-(y-a)^{2}\right)}-1\right) \\
& \leq-\varepsilon \alpha e^{-\alpha a^{2}} x\left(a^{2}-x^{2}-(y-a)^{2}\right)=-\varepsilon \alpha e^{-\alpha a^{2}} x\left(2 a y-x^{2}-y^{2}\right) \\
& \leq-a \varepsilon \alpha e^{-\alpha a^{2}} x y \quad \text { for } 0<x \leq y<\tilde{a} .
\end{aligned}
$$

Now exchanging the roles of $x, y$ and noticing that the assumptions (11.2) are symmetric in $x, y$, the conclusion already obtained guarantees that also $z\left(x, y, t_{1}\right) \leq-a \varepsilon \alpha e^{-\alpha a^{2}} x y$ for $0<y \leq x<\tilde{a}$, hence (11.4) in $(0, \tilde{a})^{2} \times\left[t_{0}, \tau_{2}\right)$. The extension to the remaining part of the rectangle $\left(0, X_{1}\right) \times\left(0, Y_{1}\right)$ (away from the corner $\left.(0,0)\right)$ follows from the Hopf boundary lemma and the strong maximum principle.

\section{References}

[1] J.M. Arrieta, A. Rodríguez-Bernal, Ph. Souplet, Boundedness of global solutions for nonlinear parabolic equations involving gradient blow-up phenomena, Ann. Scuola. Norm. Super. Pisa, Cl. Sci. (5) 3 (2004), 1-15. 
[2] A. Attouchi, Well-posedness and gradient blow-up estimate near the boundary for a Hamilton-Jacobi equation with degenerate diffusion, J. Differential Equations 253 (2012), 2474-2492.

[3] A. Attouchi, Boundedness of global solutions of a p-Laplacian evolution equation with a nonlinear gradient term, preprint (2013).

[4] G. Barles, Ph. Laurençot, C. Stinner, Convergence to steady states for radially symmetric solutions to a quasilinear degenerate diffusive Hamilton-Jacobi equation, Asymptotic Analysis 67 (2010), 229-250.

[5] J.-Ph Bartier, Ph. Laurençot, Gradient estimates for a degenerate parabolic equation with gradient absorption and applications, Journal of Functional Analysis 254 (2008), 851-878.

[6] A. Friedman, J.B. McLeod, Blow-up of positive solutions of semilinear heat equations, Indiana Univ. Math. J. 34 (1985), 425-447.

[7] A. Fujii, M. Ohta, Asymptotic behavior of blowup solutions of a parabolic equation with the p-Laplacian, Publications of the Research Institute for Mathematical Sciences 32 (1996), 503-515.

[8] V. Galaktionov, S. Posashkov, Single point blow-up for N-dimensional quasilinear equations with gradient diffusion and source, Indiana Univ. Math. J., 40 (1991), 1041-1060.

[9] Y. Giga, R.V. Kohn, Nondegeneracy of blowup for semilinear heat equations, Comm. Pure Appl. Math. 42 (1989), 845-884.

[10] J. Guo, B. Hu, Blow-up rate estimates for the heat equation with a nonlinear gradient source term, Discrete and continuous dynamical systems 20 (2008), 927-937.

[11] M. Kardar, G. Parisi, Y.C. Zhang, Dynamic scaling of growing interfaces, Phys. Rev. Lett. 56 (1986), 889-892.

[12] J. Krug, H. Spohn, Universality classes for deterministic surface growth, Phys. Rev. A 38 (1988) 4271-4283.

[13] O.A. Ladyzenskaja, V.A. Solonnikov, N.N. Ural'ceva, Linear and quasi-linear equations of parabolic type, Amer. Math. Society, 1968.

[14] Ph. Laurençot, Non-diffusive large time behavior for a degenerate viscous HamiltonJacobi equation, Comm. Partial Differential Equations 34 (2009) 281-304.

[15] Ph. Laurençot, C. Stinner, Convergence to separate variables solutions for a degenerate parabolic equation with gradient source, J. Dynamics Differential Equations 24 (2012), 29-49

[16] Ph. Laurençot, J.L. Vázquez, Localized non-diffusive asymptotic patterns for nonlinear parabolic equations with gradient absorption, Journal of Dynamics and Differential Equations 19 (2007), 985-1005. 
[17] Y.-X. Li, Ph. Souplet, Single-point gradient blow-up on the boundary for diffusive Hamilton-Jacobi equations in planar domains, Communications in Mathematical Physics 293 (2010), 499-517.

[18] G.M. Lieberman, Second order parabolic differential equations. World Scientific, Singapore, 1996.

[19] M.H. Protter, H.F. Weinberger, Maximum principles in differential equations. Corrected reprint of the 1967 original. Springer-Verlag, New York, 1984.

[20] P. Quittner, Ph. Souplet, Superlinear parabolic problems: Blow-up, global existence and steady states, Birkhauser Advanced Texts, 2007.

[21] A.A. Samarskii, V.A. Galaktionov, S.P. Kurdyumov, A.P. Mikhailov Blow-up in quasilinear parabolic equations, Nauka, Moscow, 1987. English translation: Walter de Gruyter, Berlin, 1995.

[22] J. Serrin, A symmetry problem in potential theory Arch. Rational Mech. Anal. 43 (1971), 304-318.

[23] Ph. Souplet, J. L. Vázquez, Stabilization towards a singular steady state with gradient blow-up for a diffusion-convection problem, Discrete Contin. Dyn. Sys 14 (2006), 221-234.

[24] Ph. Souplet, Q.S. Zhang, Global solutions of inhomogeneous Hamilton-Jacobi equations, J. Anal. Math. 99 (2006), 355-396.

[25] C. Stinner, Convergence to steady states in a viscous Hamilton-Jacobi equation with degenerate diffusion, Journal of Differential Equations 248 (2010), 209-228.

[26] Z. Zhang, Y.-Y. Li, Boundedness of Global Solutions for a Heat Equation with Exponential Gradient Source, Abstract and Applied Analysis 2012 (2012), 1-10.

[27] Z. Zhang, Gradient blowup rate for a viscous Hamilton-Jacobi equation with degenerate diffusion, Arch. Math. 100 (2013), 361-367.

[28] J. Zhao, Existence and nonexistence of solutions for $u_{t}=\operatorname{div}\left(|\nabla u|^{p-2} \nabla u\right)+$ $f(\nabla u, u, x, t)$, Journal of Mathematical Analysis and Applications 172 (1993), 130146.

Université Paris 13, Sorbonne Paris Cité,

Laboratoire Analyse, Géométrie et Applications, CNRS (UMR 7539),

93430 Villetaneuse, France.

E-mail: attouchi@math.univ-paris13.fr, souplet@math.univ-paris13.fr 